\author{
Banco de México \\ Documentos de Investigación
}

Banco de México

Working Papers

N॰2009-01

\title{
Pronóstico de la Volatilidad del Tipo de Cambio: el Desempeño Superior de Combinaciones Condicionales de Pronósticos de Series de Tiempo y Pronósticos Implícitos en Opciones
}

\section{Guillermo Benavides}

Banco de México
Carlos Capistrán

Banco de México

Enero 2009

La serie de Documentos de Investigación del Banco de México divulga resultados preliminares de trabajos de investigación económica realizados en el Banco de México con la finalidad de propiciar el intercambio y debate de ideas. El contenido de los Documentos de Investigación, así como las conclusiones que de ellos se derivan, son responsabilidad exclusiva de los autores y no reflejan necesariamente las del Banco de México.

The Working Papers series of Banco de México disseminates preliminary results of economic research conducted at Banco de México in order to promote the exchange and debate of ideas. The views and conclusions presented in the Working Papers are exclusively the responsibility of the authors and do not necessarily reflect those of Banco de México. 
Documento de Investigación

2009-01
Working Paper

2009-01

\title{
Pronóstico de la Volatilidad del Tipo de Cambio: el Desempeño Superior de Combinaciones Condicionales de Pronósticos de Series de Tiempo y Pronósticos Implícitos en Opciones*
}

\author{
Guillermo Benavides ${ }^{\dagger}$ \\ Carlos Capistrán \\ Banco de México \\ Banco de México
}

Resumen: Este documento provee evidencia empírica de que combinaciones de pronósticos de volatilidad implícitos en opciones y pronósticos de series de tiempo condicionadas en información actual son estadísticamente superiores a modelos individuales, combinaciones no condicionales, y pronósticos híbridos. El desempeño superior en términos de pronóstico se obtiene tanto por tomar en cuenta el desempeño esperado de cada modelo individual condicionado en información actual, como por combinar los modelos individuales. El método utilizado en este documento para producir las combinaciones condicionales extiende la aplicación de pruebas condicionales de habilidad de predicción a la selección de combinaciones de pronósticos. La aplicación es para pronósticos de volatilidad del tipo de cambio Peso Mexicano - Dólar Estadounidense, donde la volatilidad realizada calculada utilizando datos intra-día es utilizada como una aproximación de la volatilidad diaria (latente).

Palabras Clave: Cambio de Régimen, Evaluación de Pronósticos, GARCH, Pronósticos Compuestos, Tipo de Cambio Peso Mexicano - Dólar Estadounidense, Volatilidad Implícita.

Abstract: This paper provides empirical evidence that combinations of option implied and time series volatility forecasts that are conditional on current information are statistically superior to individual models, unconditional combinations, and hybrid forecasts. Superior forecasting performance is achieved by both, taking into account the conditional expected performance of each model given current information, and combining individual forecasts. The method used in this paper to produce conditional combinations extends the application of conditional predictive ability tests to select forecast combinations. The application is for volatility forecasts of the Mexican Peso-US Dollar exchange rate, where realized volatility calculated using intra-day data is used as a proxy for the (latent) daily volatility.

Keywords: Composite Forecasts, Forecast Evaluation, GARCH, Implied volatility, Mexican Peso - U.S. Dollar Exchange Rate, Regime-Switching.

JEL Classification: C22, C52, C53, G10.

\footnotetext{
*Agradecemos a Alejandro Díaz de León, Antonio E. Noriega, Carla Ysusi y a los participantes de los seminarios: Sociedad de Econometría de América Latina (LAMES) 2008 en Río de Janeiro, XII Reunión de la Red de Investigadores de Bancos Centrales del Continente Americano (CEMLA) en el Banco de España, Reunión de la Sociedad de Dinámica No Lineal y Econometría en el Banco de la Reserva Federal de San Francisco, Banco de México, ITAM, ITESM CCM, y a la Universidad del Valle de México por sus valiosos comentarios. También agradecemos a Antonio Sibaja y a Pablo Bravo por su ayuda para los datos intra-día del tipo de cambio. Andrea San Martín, Gabriel López-Moctezuma, Luis Adrián Muñiz y Carlos Muñoz Hink proporcionaron una excelente ayuda en la investigación.

$\dagger$ Dirección General de Investigación Económica. Email: gbenavid@banxico.org.mx

¥ Dirección General de Investigación Económica. Email: ccapistran@banxico.org.mx
} 


\section{Introducción}

Los pronósticos de volatilidad son fundamentales en varias aplicaciones financieras. Por ejemplo, la información sobre la volatilidad se utiliza ampliamente para la optimización del portafolio de inversión, la cobertura, la administración de riesgos, la valoración de opciones y otros tipos de derivados (Taylor, 2005). Dado que la volatilidad financiera es una medida de riesgo, los agentes de política muchas veces consideran la volatilidad para tener una idea sobre las vulnerabilidades de los mercados financieros y la economía (Poon y Granger, 2003). Asimismo, muchas decisiones se toman anticipando lo que pudiera ocurrir en el futuro. Por lo tanto, el pronóstico de volatilidad de variables financieras constituye una fuente de información importante.

Básicamente existen dos clases de modelos para pronosticar la volatilidad: los modelos basados en series de tiempo y los modelos basados en opciones (Poon y Granger, 2003). Entre los modelos de series de tiempo existen modelos basados en la volatilidad pasada, como los promedios históricos de rendimientos de precios al cuadrado; los modelos autorregresivos con heteroscedasticidad condicional (tipo ARCH), como los modelos ARCH, GARCH y EGARCH; y, los modelos de volatilidad estocástica. Entre los modelos de volatilidad basados en opciones, típicamente denominados volatilidades implícitas en opciones (IV, por sus siglas en inglés), existen los modelos del tipo Black-Scholes (Black y Scholes, 1973), una técnica sin modelo y los que se basan en datos duros de negociación de volatilidad. ${ }^{1}$

Aunque los académicos e investigadores utilizan ampliamente varios modelos para pronosticar la volatilidad, actualmente no existe consenso sobre cuál modelo es superior en términos de certeza predictiva (Poon y Granger, 2003; Taylor, 2005; Andersen et al., 2006). ${ }^{2}$ Algunos autores opinan que los modelos de series de tiempo de pronóstico de la volatilidad son superiores porque están diseñados específicamente para capturar la persistencia de la volatilidad, que es una característica típica de la volatilidad financiera (Engle, 1982; Bollerslev, 1986). Otros autores consideran que la volatilidad implícita es informativamente superior a los pronósticos de modelos de series de tiempo porque es el pronóstico del "mercado" y que, por lo tanto, puede basarse en información más amplia y además contener un componente direccionado hacia delante (forward looking) (Xu y Taylor, 1995).

En parte, esto ha llevado a investigadores a sugerir que la combinación de varios pronósticos de volatilidad es preferible. Patton y Sheppard (2007) y Anderson et al. (2006), entre

\footnotetext{
${ }^{1}$ En los mercados de opciones extrabursátiles de tipo de cambio, los inversionistas frecuentemente negocian volatilidades implícitas. Por tal motivo es que existe disponibilidad de datos duros de volatilidades implícitas.

${ }^{2}$ Se han sugerido otros métodos para pronosticar la volatilidad financiera, tales como los no paramétricos, las redes neuronales, la programación genética y los modelos basados en cambios de tiempo y duración. Sin embargo, se encontró que dichos métodos tienen relativamente menor poder predictivo y que el número de publicaciones que los utilizan como referencia es sustancialmente inferior.
} 
otros, subrayan la importancia de analizar más en detalle las especificaciones compuestas de los pronósticos de volatilidad. Becker y Clements (2008) muestran que los pronósticos compuestos de volatilidad de S\&P 500 son estadísticamente superiores a los pronósticos individuales. Pong et al. (2004) y Benavides (2006) explican que las combinaciones de pronósticos que están direccionados hacia atrás (backward looking) y hacia delante (forward looking) también pueden utilizarse exitosamente para pronosticar la volatilidad del tipo de cambio, ya que son intuitivamente atractivos porque pueden presentar dinámicas distintas. Por lo tanto, la combinación de los dos pronósticos puede ser útil para incorporar diferentes características en un sólo pronóstico, con el fin de obtener un pronóstico más realista de la volatilidad de un activo financiero. Adicionalmente, la combinación de pronósticos ha tenido una historia respetable en pronosticar otras variables económicas y financieras (Timmermann, 2006).

Existe cierta evidencia en la literatura sobre combinaciones, específicamente para pronosticar variables macroeconómicas, en donde los esquemas de combinación que cambian en el tiempo y que incluyen información actual y pasada, pueden superar a las combinaciones lineales (Deutch et al., 1994; Elliot y Timmermann, 2005; Aiolfi y Timmermann, 2006; Guidolin y Timmermann, 2006). Este tipo de combinación de pronósticos que cambia en el tiempo puede ser apropiado para pronosticar la volatilidad financiera a través del efecto de acumulamiento (clustering) de la volatilidad observada. Si se captura bien la dinámica de alta volatilidad a través de un método (o un conjunto de métodos), al mismo tiempo que se captura la dinámica de baja volatilidad mediante otro método (o conjunto de métodos), entonces una combinación de pronósticos que cambia en el tiempo pudiera ser la herramienta apropiada para aprovechar sus ventajas comparativas. Esto pudiera ser cierto, incluso aunque uno de los métodos pareciera tener una ventaja absoluta o una desventaja absoluta. En este sentido, Poon y Granger (2003) y Patton y Sheppard (2007) sugirieron que debería analizarse más este tema, considerando que casi no existen investigaciones publicadas.

Dos combinaciones específicas que cambian en el tiempo llamaron nuestra atención: una combinación propuesta por Deutch et al. (1994) con ponderaciones que cambian en el tiempo de manera discreta, y el pronóstico "híbrido" de Giacomini y White (2006) (GW), el cual elige los mejores pronósticos de manera recursiva. En la metodología de Deutch et al. (1994) se utilizan las ponderaciones apropiadas de la combinación, considerando una función indicador que muestra el régimen futuro, aunque dichos autores no proponen un método para obtener la función indicador. En contraste, GW proponen una técnica basada en una prueba condicional de habilidad de predicción (CPA, por sus siglas en inglés) para diagnosticar si la información actual es adecuada para determinar el modelo de pronóstico más certero en un momento específico en el futuro. GW exploran las implicaciones de la selección del modelo de incorporar una perspectiva condicional a través de un ejemplo simple de una regla de decisión de dos 
pasos, la cual prueba si los pronósticos que compiten tienen el mismo desempeño. En caso de rechazo, se utiliza la información actual disponible para seleccionar el mejor pronóstico para la fecha de interés en el futuro.

En el presente documento mostramos que se puede extender el procedimiento de dos pasos de GW para seleccionar combinaciones de pronóstico. Esto resulta en una combinación condicional como propuesta por Deutch et al. (1994), pero con la ventaja de que se puede probar si la información actual puede ser útil para seleccionar el régimen a futuro. La extensión del modelo es simple, porque incluye el uso de combinaciones incondicionales en el segundo paso.

Para evaluar nuestra metodología propuesta, primero evaluamos la certeza del pronóstico de algunos métodos comúnmente aplicados para pronosticar la volatilidad financiera, así como las combinaciones de éstos, utilizando datos del tipo de cambio peso mexicano (MXN)dólar estadounidense (USD). Los métodos aplicados en el presente análisis son: 1) modelo univariado general autorregresivo con heteroscedasticidad condicional (GARCH; Bollerslev, 1986, Taylor, 1986); 2) volatilidades implícitas en base al modelo obtenidas mediante el procedimiento de Garman y Kohlhagen (1983); 3) datos duros de volatilidades implícitas de cotizaciones registradas en las negociaciones en deltas específicas de opciones extrabursátiles de tipo de cambio; 4) combinaciones lineales de pronósticos de los modelos mencionados anteriormente; y, 5) combinaciones que cambian en el tiempo, conocidas como "combinaciones condicionales". 3

Nuestros resultados indican que las combinaciones condicionales de pronósticos GARCH y los pronósticos de (datos duros) de IV del tipo de cambio presentan una certeza estadísticamente superior fuera de la muestra, medida en términos de Errores de Pronóstico Cuadráticos Medios (EPCM). Estas combinaciones que cambian en el tiempo tienen ponderaciones que varían de acuerdo con los niveles pasados de volatilidad, considerando que IV tiende a mostrar un mejor desempeño cuando el nivel de volatilidad realizada es alto; mientras que los modelos GARCH tienden a ser relativamente más certeros cuando el nivel de volatilidad es bajo. Por lo tanto, nuestra combinación condicional propuesta considera la ventaja comparativa de cada uno de los dos métodos de pronóstico, el método direccionado hacia delante (forward looking) y hacia atrás (backward looking).

El presente estudio se realizó utilizando datos intra-día del tipo de cambio MXN-USD para construir una proxy de volatilidad ex-post para utilizarla como punto de referencia para evaluar los pronósticos. Como muestran Andersen y Bollerslev (1998), los datos intra-día pueden utilizarse para formar proxies ex-post de la volatilidad (latente) diaria más certeras

\footnotetext{
${ }^{3}$ Para evidencia acerca del desempeño de algunos de estos métodos para el caso de la volatilidad del tipo de cambio peso-dólar utilizando datos diarios, ver Benavides (2006).
} 
y significativas que las calculadas con datos diarios. Por lo que sabemos, los datos de alta frecuencia para el tipo de cambio específico no se han analizado en ningún otro lado en términos de pronóstico de volatilidad.

El presente documento está estructurado de la siguiente manera: en la Sección 2 se discute la metodología utilizada para obtener los pronósticos individuales, para combinarlos de manera lineal y no lineal, y para evaluar el desempeño del pronóstico. En la Sección 3 se presentan los datos y las proxies de volatilidad ex-post. En la Sección 4 se muestran los resultados empíricos y finalmente en la Sección 5 se presentan las conclusiones.

\section{Metodología}

\subsection{Modelos individuales}

Los pronósticos estimados a través de los modelos individuales descritos en el presente documento son pronósticos de un paso hacia delante (es decir, de un día de negociación hacia delante). A continuación se describe en detalle cada modelo utilizado.

\subsubsection{Modelos tipo ARCH}

La primera clase de modelos incluye los modelos tipo ARCH. Está ampliamente documentado que estos modelos pueden generar estimaciones certeras de la volatilidad de precios de activos, porque capturan los cambios a través del tiempo así como el acumulamiento (clustering) comúnmente observado en la volatilidad de los datos financieros. Varios estudios mostraron la superioridad de predicción fuera de la muestra de los modelos tipo ARCH en comparación con los modelos históricos. ${ }^{4}$ Para el caso de la volatilidad del tipo de cambio existen otros estudios como el de Cumby et al., (1993), Guo (1996), y Andersen y Bollerslev (1998), entre otros. Los resultados son mixtos para otros tipos de activos. Sin embargo, la mayoría de ellos muestra que los modelos tipo $\mathrm{ARCH}$ tienen un mejor desempeño que los modelos históricos. ${ }^{5}$ Gran parte de la literatura muestra los modelos tipo ARCH desde un punto de vista de certeza predictiva. No obstante, existe menos evidencia que este tipo de modelos aumenta el contenido de información en el análisis de regresión simple. Por ejemplo, Manfredo et al. (2001) observan que, en el contexto de regresión de Mincer-Zarnowitz (MZ), el poder explicativo de los pronósticos fuera de la muestra es relativamente bajo. En la mayoría de los casos, el coeficiente de determinación, $R^{2}$, es menor a $10 \%$. Sin embargo,

\footnotetext{
${ }^{4}$ Excluyendo los modelos ARFIMA de los modelos históricos.

${ }^{5}$ Ver, entre otros, Taylor (1986) para futuros de tasa de interés, Akgiray (1989) para acciones y Manfredo et al. (2001) para commodities agrícolas.
} 
los estudios recientes han mostrado que el uso de medidas de volatilidad realizada ex-post basadas en datos intra-día mejoran el poder explicativo en este tipo de regresiones. Se observó que el $R^{2}$ aumenta alrededor de tres veces en comparación con las medidas de volatilidad basadas exclusivamente en datos diarios (Andersen et al., 2006).

En particular, en el presente trabajo aplicamos un modelo $\operatorname{GARCH}(1,1)$ univariado. ${ }^{6}$ Este modelo parsimonioso fue elegido dentro de la familia $\mathrm{ARCH}$, de acuerdo con la evidencia presentada por Hansen y Lunde (2005) en una comparación de pronósticos entre 330 modelos tipo $\mathrm{ARCH}$, no encuentran evidencia de que un modelo haya superado al modelo GARCH(1,1) en su análisis de tipos de cambio. ${ }^{7}$ Adicionalmente, no se consideraron modelos asimétricos de volatilidad (EGARCH, GJR, QGARCH, entre otros) porque, en teoría, no son justificables para modelar la volatilidad del tipo de cambio, debido a la falta de evidencia estadística comprobada que las volatilidades del tipo de cambio son asimétricas (Engle y Ng, 1993). ${ }^{8}$ Finalmente, no se analizaron modelos $\mathrm{ARCH}$ fraccionalmente integrados debido a una limitación importante: en algunas ocasiones generan una tendencia en el tiempo en la volatilidad; sin embargo, usualmente no se observan tendencias en el tiempo en la volatilidad (Granger, 2003).

Las fórmulas para el modelo $\operatorname{GARCH}(1,1)$ son las siguientes (Andersen et al., 2006). La primera es:

$$
\begin{aligned}
& r_{t}=\mu_{t \mid t-1}+\sigma_{t \mid t-1} z_{t} \\
& z_{t} \sim i . i . d ., E\left(z_{t}\right)=0, \operatorname{Var}\left(z_{t}\right)=1,
\end{aligned}
$$

donde $r_{t}$ es el rendimiento en tiempo $t$, calculado como la diferencia logarítmica del tipo de cambio, es decir $r_{t}=p_{t}-p_{t-1}$, donde $p$ es el logaritmo natural del tipo de cambio, $\mu_{t \mid t-1}$ es la media condicional, $\sigma_{t \mid t-1}$ es la varianza condicional, y $z_{t}$ denota un proceso de errores sin correlaciones seriales, con media cero y varianza uno. El modelo $\operatorname{GARCH}(1,1)$ para la varianza condicional es:

$$
\sigma_{t \mid t-1}^{2}=\omega+\alpha \varepsilon_{t-1}^{2}+\beta \sigma_{t-1 \mid t-2}^{2}
$$

donde $\varepsilon_{t} \equiv \sigma_{t \mid t-1} z_{t}$ y los parámetros son restringidos para asegurar que la varianza condi-

\footnotetext{
${ }^{6}$ Se realizaron pruebas de ARCH-LM, siguiendo la metodología de Engle (1982), para corroborar que la serie de investigación tiene efectos $\mathrm{ARCH}$. Los resultados indican que la serie rechaza la hipótesis nula a favor de los efectos ARCH. El procedimiento se realizó utilizando hasta siete rezagos.

${ }^{7}$ En adición, se realizó una búsqueda de especificación para el orden de rezagos, utilizando criterios de información. Se aplicaron Criterios de Información Akaike (CIA) y Criterios de Información Bayesiana Schwartz (CIB). En ambos casos se seleccionó el modelo GARCH(1,1).

${ }^{8}$ No se observaron volatilidades asimétricas en los datos de este estudio. Se realizaron dos pruebas para comprobar la existencia de volatilidad asimétrica: el método de Engle y Ng (1993) y un análisis de correlaciones cruzadas de residuos estandarizados al cuadrado vs. residuos no cuadrados.
} 
cional es positiva, $\omega>0, \alpha \geqslant 0, \beta \geqslant 0$. Utilizamos una distribución-t para el proceso de error.

El modelo GARCH $(1,1)$ se estima aplicando el procedimiento estándar, explicado en Bollerslev (1986) y Taylor (1986), pero utilizando ventanas móviles. Se utilizaron dos ventanas móviles de tamaño fijo. Una contiene 756 observaciones (aproximadamente tres años de datos) y la otra, 1526 (aproximadamente seis años de datos). La ventana con tamaño fijo fue elegida considerando que seis años de datos diarios son suficientes para capturar la dinámica de volatilidad de las series que tienen coeficientes robustos con errores estándares relativamente pequeños. ${ }^{9}$ No se utilizó la estimación recursiva porque la prueba condicional de habilidad de predicción a utilizarse posteriormente no se puede aplicar cuando los pronósticos se obtienen a través de ventanas que se expanden (Giacomini y White (2006) excluyen esquemas de pronósticos con ventanas que se expanden mediante supuestos). Se estimaron los parámetros, incluyendo los grados de libertad de la distribución-t, utilizando la metodología de máxima verosimilitud y aplicando el procedimiento de Marquandt. ${ }^{10}$

\subsubsection{Modelos implícitos en opciones}

Es ampliamente conocido que la volatilidad implícita (IV, por sus siglas en inglés) en los precios de opciones es un estimador certero de la volatilidad del precio del activo subyacente (Fleming, 1998; Blair, Poon y Taylor, 2001; Taylor, 2005). Debido a que está direccionada hacia delante, la IV es intuitivamente atractiva y teóricamente distinta en comparación con los modelos históricos tipo ARCH de volatilidad condicional y direccionados hacia atrás (backward looking).

En la literatura académica existe evidencia de que el contenido de información de la IV estimada en opciones pudiera superar el de los pronósticos de series de tiempo (ver, entre otros, Fleming, Ostdiek y Whaley (1995) para índices accionarios en mercados de futuros; Fleming (1998), Blair, Poon y Taylor (2001) para acciones; Ederington y Guan (2002) para opciones sobre futuros del S\&P 500; y Manfredo, Leuthold y Irwin (2001) y Benavides (2003) para commodities agrícolas). Por otro lado, algunos documentos de investigación son escépticos respecto a la certeza de los pronósticos fuera de la muestra (ver, entre otros, Day y Lewis, 1992; Canina y Figlewski, 1993; y Lamoureux y Lastrapes, 1993).

En términos del pronóstico de volatilidad del tipo del cambio, la mayoría de la literatura documenta que la IV tiene mayor certeza así como mayor contenido de información. Los

\footnotetext{
${ }^{9}$ Nótese que actualmente no existe consenso sobre el método que debe utilizarse para determinar el tamaño óptimo de la ventana móvil (Pesaran y Timmermann, 2007).

${ }^{10}$ Dada la amplia literatura sobre este tipo de modelos, no se proporciona información más detallada. Si el lector requiere más detalles, puede consultar Bollerslev et al. (1994). En el presente documento, estos cálculos se generaron mediante la herramienta UCSD GARCH de Matlab, disponible en www.kevinsheppard.com
} 
estudios de Jorion (1995), Szakmary et al. (2003), Pong et al. (2004), y Benavides (2006) corroboran la evidencia. ${ }^{11}$ Aunque no existe consenso sobre el método que pronostica con mayor certeza, para el pronóstico de volatilidad del tipo de cambio la IV tiende a mostrar un mejor desempeño en comparación con los otros modelos (Poon y Granger, 2003).

La IV en opciones de un activo subyacente es el pronóstico de volatilidad del mercado de dicho activo durante la vida de la opción. La IV se obtiene implícitamente a través de las opciones escritas en el referido activo subyacente. En el presente documento se utilizan dos tipos de IV: uno proviene de las cotizaciones de los precios expresadas como IV, que corresponden a la delta de una opción; y otro que se basa en un modelo.

Considerando que la volatilidad es la única variable no observable en un modelo de valuación de opción, los negociadores en los mercados de opciones extrabursátiles negocian las cotizaciones de volatilidad. Esta medida presenta datos duros de la IV, normalmente utilizados por los negociadores de opciones extrabursátiles del tipo de cambio. Por mutuo acuerdo, una vez que los negociadores deciden sobre la cotización de la IV para la transacción, ésta se sustituye en un modelo de fijación de precios de opciones (que, por lo general, es el modelo de Garman y Kohlhagen (GK), 1983) para determinar el precio de las opciones en términos monetarios. La cifra final se obtiene en unidades monetarias. Por lo tanto, la compra y venta se realiza en efectivo, aunque la negociación original se haya acordado en porcentajes anualizados de volatilidad.

Para calcular la volatilidad implícita en opciones en base al modelo para un activo se requiere un modelo de valuación de opciones así como sus componentes. Los componentes típicos de un modelo de valuación de opciones monetarias son: la tasa de interés nacional libre de riesgo, $i$; la tasa de interés externa libre de riesgo, $i_{f}$; el plazo de vencimiento, $T$; el precio spot de activo subyacente, $S$; el precio de ejercicio, $X$; y el precio de mercado de la opción. En el presente estudio se utiliza el modelo GK de fijación de precios de opciones. Este modelo se deriva de la fórmula de Black-Scholes y comúnmente se utiliza en la literatura para valorar las opciones de monedas.

\footnotetext{
${ }^{11}$ Pong et al. (2004) muestran que los pronósticos de volatilidad implícita tuvieron un desempeño por lo menos igual al de los modelos históricos de memoria larga, específicamente los Modelos Autorregresivos Integrados Fraccionalmente de Media Móvil, para horizontes de uno y tres meses.
} 
El modelo GK es el siguiente:

$$
\begin{aligned}
c(X, T) & =S e^{-i_{f} T} N\left(d_{1}\right)-X e^{-i T} N\left(d_{2}\right), \\
p(X, T) & =X e^{-i T} N\left(-d_{2}\right)-S e^{-i_{f} T} N\left(-d_{1}\right), \\
d_{1} & =\frac{\ln \left(\frac{S}{X}\right)+\left(i-i_{f}+\frac{1}{2} \sigma^{2}\right) T}{\sigma \sqrt{T}}, \\
d_{2} & =d_{1}-\sigma \sqrt{T}
\end{aligned}
$$

donde $c$ es el valor de opciones de compra (call options) de estilo europeo, $p$ es el valor de opciones de venta (put options) de estilo europeo, y $N(x)$ es la función de distribución acumulada de probabilidad, que es gaussiana. $\sigma^{2}$ es la varianza anualizada de los rendimientos de precios. La volatilidad implícita se calcula mediante una técnica de inducción hacia atrás que resuelve para la única variable no observable, $\sigma^{2}$, en la función de precios de la opción de compra. ${ }^{12}$ Para cada día de negociación, dichas volatilidades implícitas se obtienen de contratos de un mes de opciones extrabursátiles para el tipo de cambio MXN-USD (cercanas a expirar). Para el presente documento se requiere un pronóstico para el próximo día de negociación y se transformó la varianza anualizada dividiéndola por 252, cifra que corresponde al número aproximado de días de negociación en un año.

\subsection{Combinaciones lineales}

Otro método utilizado para pronosticar la volatilidad financiera es la combinación de distintos modelos de pronóstico que derivan en un pronóstico compuesto para obtener pronósticos más certeros. La motivación para usar un enfoque compuesto está principalmente relacionada con los errores de pronóstico. Normalmente se observa que los errores de los modelos de pronóstico individual no tienen una correlación perfecta. En un enfoque compuesto se asume que cada modelo agrega información significativa a todo el modelo, dado que la diferencia estadística observada en los errores estimados no está perfectamente correlacionada. De igual manera, es bien conocido que se puede reducir considerablemente la varianza de errores fuera de la muestra con modelos de pronósticos compuestos (Clemen, 1989; Timmermann, 2006).

Los enfoques de pronóstico compuestos empezaron a considerarse de manera formal a finales de los años sesenta, a raíz del trabajo de Bates y Granger (1969). Clemen (1989) y Timmermann (2006) realizan una revisión de la extensa literatura que en la actualidad existe sobre este tema. Sin embargo, su uso para pronosticar la volatilidad ha sido menos frecuente.

\footnotetext{
${ }^{12} \mathrm{El}$ cálculo se realizó iterativamente hasta que el error de la fijación de precios fue menor a 0.00001. Los cálculos se realizaron utilizando Visual Basic for Applications.
} 
La mayoría de las investigaciones sobre modelos compuestos que existen en la literatura recomienda la aplicación de combinaciones lineales. Granger y Ramanathan (1984) consideran tres regresiones (la notación original se cambió para adaptarla a nuestro contexto):

$$
\begin{aligned}
& (G R 1) \tilde{\sigma}_{t+1}^{2}=\omega_{0}+\boldsymbol{\omega}^{\prime} \widehat{\boldsymbol{\sigma}}_{t+1 \mid t}^{2}+\varepsilon_{t+1} \\
& (G R 2) \tilde{\sigma}_{t+1}^{2}=\boldsymbol{\omega}^{\prime} \widehat{\boldsymbol{\sigma}}_{t+1 \mid t}^{2}+\varepsilon_{t+1} \\
& (G R 3) \tilde{\sigma}_{t+1}^{2}=\boldsymbol{\omega}^{\prime} \widehat{\boldsymbol{\sigma}}_{t+1 \mid t}^{2}+\varepsilon_{t+1}, \text { s.a. } \boldsymbol{\omega}_{t}^{\prime} \boldsymbol{\iota}=1,
\end{aligned}
$$

donde $\tilde{\sigma}_{t+1}^{2}$ se refiere a cierto tipo de medida de volatilidad ex-post, $\widehat{\boldsymbol{\sigma}}_{t+1 \mid t}^{2}$ es un vector- $K$ de pronósticos de volatilidad ex-ante, y $\iota$ es un $K \times 1$ vector de unos. La primera y segunda regresión se puede estimar mediante Mínimos Cuadrados Ordinarios (MCO) estándares, con la diferencia de que la segunda regresión omite un término de intersección. La tercera regresión omite una intersección y se puede estimar mediante Mínimos Cuadrados restringidos. La tercera especificación está motivada por el supuesto de insesgamiento de pronósticos individuales. Considerando que las ponderaciones suman uno, el pronóstico compuesto también es insesgado. ${ }^{13}$ El procedimiento propuesto por Granger y Ramanathan presenta dos características deseables: primero, genera un pronóstico compuesto que usualmente es mejor que cualquiera de los pronósticos individuales y, segundo, es un método fácil de instrumentar.

En el presente trabajo se utilizan las tres regresiones y se estiman de manera recursiva. Adicionalmente se ha encontrado que en el caso de algunas variables, una combinación lineal con ponderaciones iguales (es decir, que simplemente generan el promedio de pronósticos) produce buenos resultados en términos de los EPCM (Timmermann, 2006). Por tal motivo, también consideramos combinaciones lineales, donde las ponderaciones equivalen a $\frac{1}{K}$ y $\omega_{0}=$ 0, en términos de la ecuación anterior.

\subsection{Combinaciones condicionales}

A veces, dependiendo de la dinámica particular de la serie a pronosticar, una combinación simple, como las consideradas anteriormente, pudiera no ser suficientemente flexible. Esto es más probable si el desempeño de cada pronóstico a combinar cambia condicionalmente con la información reciente y pasada.

En este contexto, Deutch et al. (1994), entre otros, indican que las combinaciones que utilizan ponderaciones que cambian en el tiempo pueden tener un mejor desempeño que las combinaciones lineales simples, porque posiblemente pueden capturar algunas de las nolinealidades presentes en los datos. Un caso simple de ponderaciones que cambian en el tiempo

\footnotetext{
${ }^{13}$ Sin embargo, debido a las limitaciones impuestas, dicha especificación pudiera ser ineficiente.
} 
es el cambio de regímenes (regime switching). Para dos regímenes puede existir un conjunto de ponderaciones de combinación para un régimen y otro para el otro régimen. Una cuestión importante es cómo distinguir entre ambos regímenes, es decir, como determinar cuándo se deben cambiar. Un enfoque en la literatura de combinaciones es el de utilizar variables observables; otro enfoque es utilizar variables latentes. El caso de las variables observables ha sido estudiado por Deutch et al. (1994), mientras que el de las variables latentes por Elliott y Timmermann (2005). ${ }^{14}$ En línea con Deutch et al. (1994), y debido a que nuestro estudio utiliza variables observables, para el mismo se estiman modelos de pronósticos compuestos con ponderaciones que cambian en el tiempo.

En particular, Deutch et al. (1994) proponen la combinación de pronósticos utilizando ponderaciones que cambian (la notación original se cambió para adaptarla a nuestro contexto):

$$
\begin{aligned}
\widehat{\sigma}_{t+1, t, c}^{2} & =I_{(\cdot)}\left(\alpha_{0}+\boldsymbol{\alpha} / \widehat{\boldsymbol{\sigma}}_{t+1 \mid t}^{2}\right) \\
& +\left(1-I_{(\cdot)}\right)\left(\beta_{0}+\boldsymbol{\beta} / \widehat{\boldsymbol{\sigma}}_{t+1 \mid t}^{2}\right),
\end{aligned}
$$

donde $\widehat{\sigma}_{t+1, t, c}^{2}$ es el pronóstico compuesto, y $I_{(\cdot)}$ es la función indicador. Se examinaron varias opciones para construir la función indicador, como por ejemplo los errores de pronósticos pasados o las variables económicas relevantes para la aplicación del pronóstico de inflación. Aunque muestran exitosamente que los métodos que cambian en el tiempo pueden reducir sustancialmente los EPCM, Deutch et. al (1994) no proponen una manera para seleccionar las variables utilizadas para determinar los regímenes (es decir, para calcular la función indicador), ni tienen una prueba para corroborar si es adecuado considerar la combinación que cambia en el tiempo. ${ }^{15}$

Cuando se trabaja con regresiones que cambian, usualmente se enfrenta el problema de encontrar la variable (o conjunto de variables) apropiada(s) para determinar un régimen. El problema se puede agravar en un contexto de pronóstico, ya que se requiere una variable que permita predecir el régimen en el futuro. En el presente documento proponemos el uso de la prueba CPA de Giacomini y White (2006) para seleccionar el régimen apropiado. Mediante esta técnica se puede comprobar si una variable o un conjunto de variables pueden predecir el pronóstico que va a tener el mejor desempeño en un periodo determinado en el futuro. Nuestro estudio utiliza esta técnica para construir la función indicador, que a su vez puede utilizarse para estimar combinaciones que cambian en el tiempo, como en Deutch et al.

\footnotetext{
${ }^{14}$ En este contexto, la variable latente se refiere a la(s) variable(s) utilizada(s) para determinar el régimen, y no se refiere al hecho de que la volatilidad en sí misma no es observable.

${ }^{15}$ Nuestro procedimiento, a desarrollarse más adelante, permite comprobar si es adecuado considerar las combinaciones que cambian en el tiempo.
} 
(1994).

Giacomini y White (2006) proponen una regla de decisión de dos pasos que utiliza información reciente para seleccionar el mejor pronóstico entre un par de pronósticos para la fecha de interés en el futuro.

En el primer paso se realiza la prueba CPA, donde la hipótesis nula es:

$$
\begin{aligned}
H_{0} & : E\left[\left(\tilde{\sigma}_{t+1}^{2}-\widehat{\sigma}_{t+1 \mid t, 1}^{2}\right)^{2}-\left(\tilde{\sigma}_{t+1}^{2}-\widehat{\sigma}_{t+1 \mid t, 2}^{2}\right)^{2} \mid \mathcal{F}_{t}\right] \\
& =E\left[e_{t+1 \mid t, 1}^{2}-e_{t+1 \mid t, 2}^{2} \mid \mathcal{F}_{t}\right]=0 \quad t=1,2, \ldots,
\end{aligned}
$$

donde $\widehat{\sigma}_{t+1 \mid t, i}^{2}$ es el pronóstico de la volatilidad ex-ante, generado mediante el modelo $i ; e_{t+1 \mid t, i}$ es el error del pronóstico del modelo $i$; y $\mathcal{F}$ denota un conjunto de información. A partir de (5), se puede derivar la siguiente condición de ortogonalidad:

$$
E\left[h_{t}\left(e_{t+1 \mid t, 1}^{2}-e_{t+1 \mid t, 2}^{2}\right)\right]=0 \text { para todas las funciones } h_{t} \text { medidas mediante } \mathcal{F}_{t} .
$$

Bajo este contexto, $h_{t}$ es la función prueba. La prueba se puede realizar utilizando la regresión fuera de la muestra:

$$
\begin{gathered}
e_{t+1 \mid t, 1}^{2}-e_{t+1 \mid t, 2}^{2}=\delta^{\prime} h_{t}+\epsilon_{t} \\
H_{0}: \delta=0 .
\end{gathered}
$$

Un rechazo se interpreta como que $h_{t}$ contiene información para predecir el pronóstico que tendrá el mejor desempeño para la fecha de interés en el futuro. Por lo tanto, el primer paso consiste en aplicar la prueba CPA de GW para comprobar si es posible encontrar una variable o un conjunto de variables que puedan predecir el desempeño futuro de cada pronóstico. El objetivo es predecir el pronóstico que tendrá la menor pérdida en el periodo siguiente.

Si la hipótesis nula se rechaza, el segundo paso de GW contiene la siguiente regla de decisión: utiliza $\widehat{\sigma}_{t+1, t, 2}^{2}$ si $\widehat{d}_{t+1 \mid t} \geq 0$ y utiliza $\widehat{\sigma}_{t+1, t, 1}^{2}$ si $\widehat{d}_{t+1 \mid t}<0$, donde $\widehat{d}_{t+1 \mid t}=\widehat{\delta}^{\prime} h_{t}$ es el diferencial de pérdida predictivo. Por lo tanto, el segundo paso utiliza la información de la prueba CPA para generar la función indicador, la cual se utiliza para distinguir entre dos regímenes. Si la pérdida diferencial indica que un pronóstico tendrá un mejor desempeño en el futuro, la función indicador selecciona dicho pronóstico. Éste es un tipo particular de combinación con ponderaciones que cambian, donde dichas ponderaciones son 0 ó 1 , y donde se utiliza una variable o un conjunto de variables para seleccionar un régimen.

En el presente documento extendemos el segundo paso de GW y proponemos la siguiente 
regla de decisión: utiliza $\alpha_{0}+\alpha_{1} \widehat{\sigma}_{t+1, t, 1}^{2}+\alpha_{2} \widehat{\sigma}_{t+1, t, 2}^{2}$ si $\widehat{d}_{t+1 \mid t} \geq 0$ y utiliza $\beta_{0}+\beta_{1} \widehat{\sigma}_{t+1, t, 1}^{2}+$ $\beta_{3} \widehat{\sigma}_{t+1, t, 2}^{2}$ si $\widehat{d}_{t+1 \mid t}<0$. Bajo este contexto, esperaríamos que $\widehat{\sigma}_{t+1, t, 2}^{2}$ reciba una ponderación relativamente mayor, cuando $\widehat{d}_{t+1 \mid t} \geq 0$ y que $\widehat{\sigma}_{t+1, t, 1}^{2}$ reciba una ponderación mayor cuando $\widehat{d}_{t+1 \mid t}<0$. Por lo tanto, no seguimos todo el procedimiento completo, como GW, para cambiar completamente de un pronóstico a otro, pero mantenemos la ventaja de combinaciones entre regímenes.

Una vez que obtenemos la función indicador del primer paso de GW, estimamos la combinación que cambia en el tiempo, utilizando el MCO en la siguiente regresión:

$$
\begin{aligned}
\tilde{\sigma}_{t+1}^{2} & =I_{\left(\widehat{d}_{t+1 \mid t} \geq 0\right)}\left(\alpha_{0}+\alpha_{1} \widehat{\sigma}_{t+1, t, 1}^{2}+\alpha_{2} \widehat{\sigma}_{t+1, t, 2}^{2}\right) \\
& +\left(1-I_{\left(\widehat{d}_{t+1 \mid t}>0\right)}\right)\left(\beta_{0}+\beta_{1} \widehat{\sigma}_{t+1, t, 1}^{2}+\beta_{2} \widehat{\sigma}_{t+1, t, 2}^{2}\right)+\varepsilon_{t+1}
\end{aligned}
$$

\subsection{Evaluación del desempeño del pronóstico}

La métrica utilizada en el presente análisis para ejecutar la prueba de certeza de predicción es la de los EPCM, calculados para cada tipo de pronóstico $i$ como:

$$
E P C M_{i}=P^{\frac{1}{2}} \sum_{t=1}^{P}\left(\tilde{\sigma}_{t}^{2}-\hat{\sigma}_{t \mid t-1, i}^{2}\right)^{2}
$$

donde $P$ es igual al número de observaciones fuera de la muestra. La elección de la función de pérdida considera su simplicidad, su uso generalizado así como que genera una clasificación consistente de modelos para proxies de volatilidad comúnmente utilizados (Patton, 2006). ${ }^{16}$

El método con los menores EPCM es el que consideramos ser el más certero para pronosticar la volatilidad. Para comparar la significancia estadística de los EPCM, aplicamos la prueba de Diebold y Mariano (1995) y West (1996) (prueba Diebold-Mariano-West).

Para evaluar la calidad de los pronósticos de volatilidad ex-ante necesitamos una medida de la volatilidad ex-post como punto de referencia (por ejemplo, para calcular errores de pronóstico y como variable dependiente en las regresiones de combinaciones). Una medida típicamente utilizada en la literatura de evaluación de pronósticos de volatilidad es el rendimiento diario al cuadrado. Sin embargo, las investigaciones recientes han mostrado que los rendimientos diarios al cuadrado utilizados como medida de la volatilidad realizada son proxies con ruido para la volatilidad diaria verdadera (latente) (Taylor, 2005; Andersen et al., 2006).

\footnotetext{
${ }^{16}$ Patton (2006) deriva condiciones necesarias y suficientes para la función de pérdida para que la clasificación de los pronósticos de volatilidad sea robusta ante la presencia de ruido en la proxy de volatilidad. En su trabajo se muestra que la pérdida de ECM es robusta. La volatilidad realizada es una de las proxies de la volatilidad utilizada en Patton (2006) para derivar este resultado.
} 
En contraste, varios documentos han destacado los beneficios de utilizar datos intradía para calcular una proxy de la volatilidad financiera. Parkinson (1980) fue uno de los primeros en proponer el uso de este tipo de datos de alta frecuencia. Garman y Klass (1980), Taylor (1986), y Andersen y Bollerslev (1998), entre otros, también coinciden. La volatilidad realizada construida con datos intra-día ha demostrado ser una proxy con menos ruido para la volatilidad diaria (latente) (Andersen y Bollerslev, 1998). El uso de datos intra-día se basa en la idea de que durante un periodo en el tiempo, la volatilidad se puede estimar más eficientemente cuando la frecuencia de los rendimientos aumenta, siempre y cuando los rendimientos intra-periodos no estén correlacionados entre sí. Sin embargo, existe un intercambio, porque una frecuencia tan alta en las cotizaciones intra-día, por ejemplo, un minuto, puede aumentar el sesgo debido a efectos de la microestructura de mercado (por ejemplo, ruido de diferencial demanda-oferta y registro de precios en tiempo discreto).

La varianza realizada utilizando datos intra-día se puede calcular como sigue:

$$
R V_{t}=\sum_{j=1}^{1 / \Delta}\left[p_{t+j \Delta}-p_{t+(j-1) \Delta}\right]^{2},
$$

con $\Delta$ pequeño, positivo, y con $1 / \Delta \gg 1$. Nótese que con $\Delta=1$ la medida anterior coincide con los rendimientos diarios al cuadrado. Andersen y Bollerslev (1998) sugieren que fijar $\Delta$ a 5 minutos es la frecuencia que favorablemente intercambia con el sesgo y la inconsistencia inducida por el ruido de microestructura y la eficiencia adquirida por el uso de frecuencias más altas. Anderson et al. (2006) destacan que la evidencia empírica actual para activos constantemente negociados indica que fijar $\Delta$ entre 5 y 15 minutos usualmente funciona bien (p.859). De hecho, tomar muestras de los precios en intervalos de cinco minutos probablemente es la elección más popular en la actualidad.

En el resto del presente documento utilizamos la volatilidad realizada, calculada como la varianza realizada utilizando los datos intra-día en intervalos de cada cinco minutos, como la proxy de la volatilidad ex-post. Por lo tanto, la evaluación del desempeño del pronóstico de modelos distintos se realizó utilizando este punto de referencia. Sin embargo, el tipo de cambio que utilizamos no se negoció tan frecuentemente como los tipos de cambio en los cuales se basa la evidencia del uso de intervalos de cinco minutos. Por tal motivo, reportaremos un ejercicio de robustez utilizando los rendimientos diarios al cuadrado como punto de referencia. 


\section{Datos}

\subsection{Rendimientos diarios e intra-diarios}

Los datos diarios del tipo de cambio spot MXN-USD corresponden a los precios diarios spot obtenidos de la página web del Banco de México. ${ }^{17}$ Dichos precios son promedios diarios de las cotizaciones ofrecidas por los bancos principales de México y otros intermediarios financieros. El periodo de la muestra comprende del 2 de enero de 1998 al 31 de diciembre de 2007 y en total incluye 2,499 observaciones.

Los datos intra-diarios son los del tipo de cambio MXN-USD con una frecuencia de cinco minutos. Las transacciones fueron realizadas a través de la plataforma electrónica de Reuters. La referencia exacta es Reuters Matching (RIC: MXN=D2). Se consideraron transacciones desde la observación 96 hasta la observación 240. Se eligió dicho intervalo porque casi $95 \%$ de las transacciones se encuentran en este rango. Los fines de semana y los días feriados se excluyeron debido a la negociación significativamente infrecuente en esos días. El tamaño de la muestra de datos intra-día es del 2 de enero de 2004 al 31 de diciembre de 2007. El tamaño de la muestra fue seleccionado considerando la disponibilidad de los datos. ${ }^{18}$ Se utilizan las observaciones de cada intervalo de cinco minutos o la última observación si no hay una observación en el intervalo exacto del tiempo.

\subsection{Volatilidad realizada}

Las medidas de volatilidad ex-post que utilizamos como variables objetivo se calculan mediante la ecuación (7). Nuestra medida preferida utiliza los datos intra-día. Sin embargo, calculamos la volatilidad realizada utilizando los datos diarios. En la Gráfica 1 se muestran las dos estimaciones para el periodo del 2 de enero de 2004 al 31 de diciembre de 2007. Se observa que las estimaciones que utilizan datos intra-día con intervalos de cinco minutos capturan relativamente bien la dinámica de la volatilidad estimada utilizando datos diarios. Sin embargo, las primeras muestran menos ruido, como reflejo de los resultados matemáticos obtenidos por Andersen y Bollerslev (1998), de que el estimador intra-día tiene una varianza menor (como $\Delta \rightarrow 0$, la varianza disminuye).

\footnotetext{
${ }^{17}$ Banco de México es el banco central de México. Su página web es http://www.banxico.org.mx.

${ }^{18}$ En nuestra base de datos faltaron 10 días de negociación (las primeras dos semanas de mayo 2004). Para estos días se utilizaron las cotizaciones diarias (publicadas por el Banco de México). Los datos faltantes representan menos de $1 \%$ del número total de las observaciones consideradas en las evaluaciones fuera de la muestra.
} 


\subsection{Volatilidad implícita}

La volatilidad implícita en datos de opciones se calcula con los datos de los contratos de opciones extrabursátiles con vencimiento a un mes del tipo de cambio diario MXN-USD. Los datos duros de IV fueron descargados de UBS - una institución financiera internacional con base en Suiza - (el código es 1MDNMXNUSDImplied). El periodo de la muestra de los datos de opciones comprende del 2 de enero de 2004 al 31 de diciembre de 2007 e incluye 993 observaciones diarias. Se obtuvieron la tasa de interés libre de riesgo de México y de EE.UU. para estimar la IV del método basado en el modelo. Las tasas de interés de los bonos a un mes del gobierno federal de México (CETES) se descargaron de la página web del Banco de México. Los Certificados de Depósito (CDs) de EE.UU. con la misma fecha de vencimiento se obtuvieron de la página web de la Junta de Gobierno de la Reserva Federal de EE.UU. ${ }^{19}$ Las IV en base al modelo se calcularon del 2 de enero de 2004 al 31 de diciembre de 2007.

\section{Resultados empíricos}

En los siguientes ejercicios, el periodo de evaluación fuera de la muestra comprende del 2 de enero de 2004 al 31 de diciembre de 2007 para los métodos individuales, y del 2 de enero de 2006 al 31 de diciembre de 2007 para todos los métodos cuando las combinaciones están incluidas en los métodos de pronóstico. Para el último caso, se utilizaron los datos de 2004 y 2005 para iniciar la estimación recursiva de las ponderaciones de la combinación.

\subsection{Modelos individuales}

La Gráfica 2 muestra los errores de pronóstico de los métodos individuales. Las abreviaturas representan los siguientes modelos: volatilidad implícita en base al modelo (ivmb), datos duros de volatilidad implícita con vencimiento a una semana (iv1week) y a tres semanas (iv3weeks), y GARCH con ventanas móviles de tres años (GARCHroll3) y con ventanas móviles de seis años (GARCHroll6) de datos diarios. La gráfica muestra que la volatilidad implícita en base al modelo no genera buenos pronósticos en comparación con los demás. Los pronósticos ivmb son muy sesgados (sistemáticamente sobreestiman la volatilidad realizada) y tienen una varianza alta.

El Cuadro 1 contiene las estadísticas descriptivas sobre el desempeño de los modelos individuales. Los errores medios negativos tienden a sobreestimar sistemáticamente la volatilidad realizada, con la IV en opciones en base al modelo mostrando un sesgo considerablemente mayor. La última columna muestra el cociente del EPCM, utilizando el EPCM del GARCH

\footnotetext{
${ }^{19}$ La página web de la Reserva Federal de EE.UU. es http://www.federalreserve.gov/
} 
con ventanas móviles de seis años como denominador común, dado que el modelo individual fue el que tuvo el mejor desempeño en términos de los EPCM. El cociente para la IV en base al modelo es extremamente alto, confirmando que, en efecto, dicho método es particularmente inapropiado para pronosticar la volatilidad realizada. En contraste, las diferencias entre los GARCH y las IV (datos duros) son menores, aparentemente con una pequeña ventaja para los GARCH. ${ }^{20}$

El Cuadro 2 muestra el estadístico-t (arriba de la diagonal) y los valores-p (abajo de la diagonal) de las pruebas Diebold-Mariano-West en pares, donde utilizamos el estimador de la matriz de covarianza con heteroscedasticidad y autocorrelación consistente con Newey y West (1987). Los bajos valores-p pueden ser interpretados como un rechazo de la hipótesis nula de igualdad predictiva. El cuadro muestra evidencia de que la IV en base al modelo es estadísticamente peor que todos los demás métodos. En un nivel de significancia de $5 \%$ se observa claramente que: (i) ambas IV de datos duros estadísticamente muestran el mismo desempeño (valor-p es 0.3711); (ii) el GARCH estimado utilizando ventanas móviles de seis años no es estadísticamente superior al GARCH con ventanas móviles de tres años (valor-p es 0.1164); y (iii) ambas IV de datos duros muestran estadísticamente el mismo desempeño que el de los dos modelos GARCH (en pares); es decir, tienen EPCM estadísticamente indistinguibles, porque las pruebas DMW de capacidad predictiva no pueden rechazar la hipótesis nula de igualdad predictiva entre los pronósticos, con excepción del par GARCHroll6 y iv1week, para lo cual existe cierta evidencia del desempeño superior para el pronóstico tipo ARCH.

Nuestros resultados confirman que, en efecto, es difícil elegir entre pronósticos generados utilizando los métodos de series de tiempo y aquéllos obtenidos del mercado financiero (de volatilidad implícita en opciones).

\subsection{Combinaciones lineales}

El Cuadro 3 presenta las estadísticas descriptivas del desempeño de las combinaciones lineales. Todas las combinaciones incluyen pronósticos de los cinco diferentes métodos reportados en el Cuadro 1. De igual manera se presentan los estadísticos del modelo GARCH con ventanas móviles de seis años como referencia, aunque en contraste con los Cuadros 1 y 2, este análisis se realizó utilizando únicamente los pronósticos de 2006 y 2007, porque los datos de 2004 y 2005 se utilizan para iniciar la estimación (recursiva) de las ponderaciones de la combinación. Se observa que incluso la combinación que contiene una constante (GR1) tiene un error medio negativo, aunque menor que los de las demás combinaciones. Después de observar

\footnotetext{
${ }^{20}$ De igual manera evaluamos el desempeño de pronósticos históricos de la volatilidad, utilizando diferentes ventanas de estimación, de 5 a 252 días. Sin embargo, en todos los casos muestran un desempeño débil, incluso en comparación con los pronósticos de la volatilidad implícita en opciones en base al modelo.
} 
los cocientes de los EPCM, las combinaciones con ponderaciones iguales no generan buenos resultados. Adicionalmente, tres de las combinaciones presentan un cociente menor a uno, que indica que sus EPCM son menores que los del mejor modelo individual (GARCHroll6) calculado con la misma muestra reducida. Esto confirma que la combinación de pronósticos es un procedimiento que mejora la certeza del pronóstico. Sin embargo, los resultados de las pruebas DMW aplicadas a las combinaciones lineales (tomando el GARCHroll6 como punto de referencia) muestran que sólo GR1 y GR2 superan al mejor modelo individual en un nivel de significancia de $5 \%$.

Es interesante que las dos mejores combinaciones son GR1, que es la menos restringida de las regresiones de Granger-Remanathan, y GR2, que es la combinación que no incluye una constante. El segundo resultado es la consecuencia de: (i) en la mayoría de las regresiones la constante es cercana a cero; y, (ii) en general, las ponderaciones estimadas para los pronósticos de GARCH son usualmente negativas (alrededor de -0.5), mientras que las ponderaciones estimadas para las IV por lo general son positivas (alrededor de 1), lo que compensa el sesgo de los pronósticos individuales. Efectivamente, los beneficios de las combinaciones resultan de una lógica similar a la de la diversificación en los portafolios de inversión. En este caso, debido al signo negativo de las ponderaciones, el mejor "portafolio" por lo general se rezaga en comparación con los modelos GARCH. La heterogeneidad en las ponderaciones también explica el desempeño débil de los pronósticos medios en este contexto y abre las puertas hacia un mejor uso de dichos pronósticos de esquemas de combinaciones que cambian en el tiempo.

\subsection{Combinaciones condicionales}

En esta sección, primero se lleva a cabo la evaluación condicional de los pronósticos individuales (primer paso en GW). Posteriormente utilizamos los resultados para formar la función indicador y después estimamos las combinaciones que cambian en el tiempo, siguiendo la ecuación (6). Finalmente, evaluamos el desempeño de los pronósticos propuestos y compuestos que cambian en el tiempo.

El Cuadro 4 presenta los resultados de la prueba de CPA para los pronósticos individuales, utilizando los pronósticos del 2 de enero de 2004 al 30 de diciembre de $2005 .^{21}$ El cuadro presenta, abajo de la diagonal, los valores-p de la prueba GW utilizando solamente una constante como instrumento. Dichas pruebas son muy similares a las de la prueba DMW, en el sentido de que pueden ser interpretadas como pruebas incondicionales. En este caso, al nivel de $1 \%$ hay cuatro hipótesis nulas que no se pueden rechazar y que correspon-

\footnotetext{
${ }^{21}$ Se excluyeron los datos de 2006 y 2007 en la evaluación fuera de la muestra.
} 
den a los pares de GARCHroll6-GARCHroll3, iv1week-GARCHroll6, iv1week-GARCHroll3, y iv3weeks-GARCHroll3. ${ }^{22}$ Estos resultados significan que, incondicionalmente, los pares tienen EPCM estadísticamente iguales. Arriba de la diagonal, el cuadro presenta los valores-p de la prueba CPA utilizando tanto valores rezagados de la volatilidad realizada como instrumento, porque esta variable se propone fácilmente por sí misma debido al acumulamiento de volatilidad (clustering), como valores rezagados del diferencial de pérdida estimada, $d_{t}$. En este caso, la hipótesis nula de igualdad de CPA se rechaza en dos de los cuatro pares, el par GARCHroll6-iv1week en un nivel de $10 \%$ y el par iv3weeks-GARCHroll3 en un nivel de $1 \%$. Dichos resultados indican que se pueden utilizar los instrumentos elegidos para pronosticar el método que será más certero el día siguiente. ${ }^{23}$

Lo relevante es saber elegir la regla apropiada así como las variables para formar la función indicador. GW utilizan un rezago de la variable a pronosticar para predecir el diferencial de pérdida en el futuro. De acuerdo con los resultados presentados en el Cuadro 4, aplicamos la siguiente regresión:

$$
d_{t \mid t-1}=\gamma_{0}+\sum_{i=1}^{k} \gamma_{i} R V_{t-i}+\sum_{j=1}^{p} \phi_{j} d_{t-j \mid t-1-j}+\varepsilon_{t}
$$

donde $d_{t \mid t-1}$ representa el diferencial de pérdida entre cada uno de los dos pares resultantes de la aplicación de la prueba CPA (por ejemplo, el GARCHroll6 y el iv1week). En contraste con GW, utilizamos rezagos de la volatilidad realizada, así como rezagos del diferencial de pérdida. Esta ecuación es básicamente una ecuación de pronóstico que, utilizando variables conocidas en $t$, se puede utilizar para pronosticar si el diferencial de pérdida será positivo o negativo en $t+1$.

Los pasos que seguimos para generar las combinaciones condicionales son:

1. En la muestra de estimación (2004 y 2005), estimamos la regresión (8), seleccionando el número de rezagos utilizando CIA. ${ }^{24}$ Los datos que corresponden a 2006 y 2007 se excluyeron de la evaluación fuera de la muestra.

2. Formamos una variable dummy de la siguiente manera:

$$
D_{t}=\left\{\begin{array}{ccc}
1 & \text { si } & \widehat{d}_{t \mid t-1} \geq 0 \\
0 & \text { si } & \widehat{d}_{t \mid t-1}<0
\end{array}\right.
$$

\footnotetext{
${ }^{22}$ La diferencia entre estos resultados y los de DMW, como muestra el Cuadro 2, se debe principalmente a las diferentes muestras aplicadas. En el Cuadro 2 la muestra es del 2 de enero de 2004 al 31 de diciembre de 2007, mientras que en el Cuadro 4 la muestra es del 2 de enero de 2004 al 30 de diciembre de 2005.

${ }^{23}$ Los resultados son robustos ante el uso de rezagos diferentes y el uso de únicamente un sub-conjunto de instrumentos.

${ }^{24}$ Los resultados son robustos ante el uso de los CIB.
} 
3. Posteriormente utilizamos la variable dummy para estimar la regresión (6) rezagada por un periodo:

$$
R V_{t}=\delta_{0}+\delta_{1} D_{t}+\delta_{2} \widehat{\sigma}_{t \mid t-1, i}^{2}+\delta_{3} D_{t} \widehat{\sigma}_{t \mid t-1, i}^{2}+\delta_{4} \widehat{\sigma}_{t \mid t-1, j}^{2}+\delta_{5} D_{t} \widehat{\sigma}_{t \mid t-1, j}^{2}+\varepsilon_{t},
$$

utilizando datos de 2004 y 2005 para el par de los pronósticos $i, j$ (por ejemplo, GARCHroll6 y iv1w).

4. Para calcular el pronóstico compuesto para el primer día de 2006 se necesita pronosticar $\widehat{d}_{t+1 \mid t}$ utilizando la regresión estimada en el paso 1 anterior, pero actualizando las variables en el lado derecho, de tal manera que las más recientes se encuentren en $t$.

5. Después, utilizamos $\widehat{d}_{t+1 \mid t}$ para definir el valor de la variable dummy $D_{t+1}$.

6. Finalmente, el pronóstico compuesto condicionalmente para el primer día de 2006 es:

$$
\widehat{\sigma}_{t+1 \mid t, c}^{2(\text { Condicional })}=\widehat{\delta}_{0}+\widehat{\delta}_{1} D_{t+1}+\widehat{\delta}_{2} \widehat{\sigma}_{t+1 \mid t, i}^{2}+\widehat{\delta}_{3} D_{t+1} \widehat{\sigma}_{t+1 \mid t, i}^{2}+\widehat{\delta}_{4} \widehat{\sigma}_{t+1 \mid t, j}^{2}+\widehat{\delta}_{5} D_{t+1} \widehat{\sigma}_{t+1 \mid t, j}^{2}
$$

7. Para calcular los pronósticos para los días siguientes (segundo día de 2006, tercer día de 2006, ..., último día disponible de 2007) repetimos los pasos del 1 al 6 pero agregando cada vez una observación (primer día de 2006, segundo día de 2006, ..., penúltimo día disponible en 2007) de manera recursiva y siempre asegurando que se utilicen observaciones hasta el tiempo $t$ para pronosticar en el tiempo $t+1$.

Un diferencial de pérdida positivo refleja periodos de alta volatilidad, mientras que un diferencial de pérdida negativo refleja periodos de baja volatilidad. La Gráfica 3 muestra que cuando separamos los rendimientos y calculamos la distribución empírica de los rendimientos correspondientes a las fechas cuando el diferencial de pérdida se pronosticó positivo, y la distribución empírica de los rendimientos correspondientes a las fechas cuando el diferencial de pérdida se pronosticó negativo, en efecto, los diferenciales de pérdida positivos están asociados con periodos de alta volatilidad.

Cuando utilizamos la función indicador para construir un pronóstico híbrido, con base en GW, la regla implica que los modelos GARCH deberían utilizarse cuando se pronostica que dicha diferencia es negativa (es decir, cuando predecimos que la función de pérdida del modelo GARCH será menor a la pérdida de la IV), mientras que los datos duros de la IV deberían utilizarse cuando es positiva. Estos resultados coinciden con lo que se puede observar en la Gráfica 4. El modelo de opción puede competir con la volatilidad realizada en los periodos de alta volatilidad, pero sobreestima durante episodios de baja volatilidad, particularmente con respecto a lo que hace el modelo GARCH. 
El Cuadro 5 presenta los cocientes de los EPCM, con respecto a los EPCM del mejor método individual, el GARCHroll6, la mejor combinación lineal, el GR1, el pronóstico híbrido y la combinación condicional propuesta en este documento (todos en pares), calculados para la misma muestra. Nótese que en contraste con los resultados en el Cuadro 3, el método de la combinación GR1 reportado en el Cuadro 5 sólo combina el par de pronósticos correspondientes. Los EPCM de las combinaciones que cambian en el tiempo son más pequeños que los de cualquier otro modelo del pronóstico considerado en el Cuadro 5. Adicionalmente, las pruebas DMW confirman que las combinaciones condicionales tienen EPCM estadísticamente superiores a los de GARCHroll6, y en uno de los dos casos son incluso estadísticamente superiores a los EPCM de la mejor combinación lineal. La conclusión general es que las combinaciones condicionales son superiores en términos de los EPCM.

Para probar la robustez, repetimos el ejercicio pero utilizamos la volatilidad diaria al cuadrado como proxy para la volatilidad diaria (latente). De acuerdo con lo esperado, dado que esta medida es más ruidosa que la que utilizó datos intra-día, los EPCM para todos los pronósticos y todas las combinaciones de pronósticos aumentaron. El ruido adicional ciertamente complica diferenciar entre desempeños de pronósticos diferentes. Sin embargo, también en este caso encontramos que los EPCM de las combinaciones condicionales son menores que los de otros modelos considerados. El Cuadro 6 muestra los resultados en el mismo formato que el Cuadro 5. Lo primero que puede apreciarse es que uno de los pares utilizados para la combinación ha cambiado en comparación con el Cuadro 5. El primer par es el del modelo GARCHroll3 e iv1w, en lugar del GARCHroll6 e iv1w. Esto es un resultado directo de las pruebas CPA (no reportadas) y del hecho que ahora el modelo individual con los EPCM más pequeños es el GARCHroll3. Sin embargo, los resultados cualitativos son iguales. Los cocientes de los EPCM de las combinaciones condicionales son los cocientes más pequeños y la prueba estadística de precisión predictiva indica que tienen un buen desempeño. Además, los pares detectados a través de las pruebas CPA siempre incluyen un miembro de cada tipo de pronósticos, lo que significa que las combinaciones condicionales consideran las ventajas comparativas de cada tipo.

\section{Resumen y conclusiones}

La discusión reciente sobre el modelo más certero para pronosticar la volatilidad de los rendimientos de precios de los activos financieros ha generado numerosos trabajos de investigación. Muchos trabajos han comparado los modelos tipo ARCH versus las volatilidades implícitas en opciones. Aunque la mayoría de la literatura recomienda el uso de volatilidades implícitas en opciones como la alternativa más certera para pronosticar las volatilidades de 
los rendimientos de precios, aún no existe consenso en términos de encontrar un modelo único y superior. Esto básicamente responde a que la evaluación estadística de los pronósticos en general ha demostrado que los modelos en competencia tienen un desempeño peor o presentan igual certeza estadística. Más recientemente y siguiendo una literatura extensa de pronósticos macroeconómicos, los pronósticos compuestos mostraron ser un método adecuado para mejorar el desempeño de los pronósticos.

En el presente documento se comparan dichos modelos de pronóstico de volatilidad para encontrar el método más certero para pronosticar la volatilidad de los rendimientos diarios spot del tipo de cambio MXN-USD. Se utilizaron los datos del tipo de cambio intra-día con intervalos de cinco minutos para construir la volatilidad realizada, que utilizamos como medida preferida de la volatilidad ex-post. Los resultados muestran que, cuando se comparan los pronósticos individuales, casi no existe evidencia para rechazar la hipótesis nula de igualdad de capacidad predictiva entre los modelos tipo ARCH y las volatilidades implícitas (datos duros). En cuanto a las combinaciones lineales, en general, los pronósticos compuestos tienen EPCM más pequeños en comparación con los pronósticos individuales.

Sin embargo, el mejor pronóstico se obtiene cuando se combina un pronóstico tipo ARCH y un pronóstico de la volatilidad implícita en opciones utilizando ponderaciones que cambian en el tiempo. Utilizando la volatilidad pasada, así como el desempeño pasado del pronóstico para predecir el régimen, una combinación condicional que asigna un peso relativamente mayor a la volatilidad implícita en opciones durante regímenes de alta volatilidad y relativamente menor peso durante regímenes de baja volatilidad, tiene un EPCM aproximadamente $50 \%$ menor que el EPCM del mejor modelo individual, y aproximadamente $25 \%$ menor que el EPCM de la mejor combinación (incondicional). Dichos resultados muestran claramente un incremento de certeza en los pronósticos.

Futuros trabajos de investigación deberían considerar las combinaciones condicionales, incluyendo los pronósticos múltiples, así como explorar la combinación condicional de los pronósticos para otras variables, como por ejemplo la inflación, la tasa de interés y la volatilidad de otros activos. 


\section{Referencias}

[1] Aiolfi, M. y Timmermann A. (2006). Persistence in Forecasting Performance and Conditional Combination Strategies. Journal of Econometrics, 135, 31-53.

[2] Andersen, T.G., Bollerslev, T., Christoffersen, P. F., y Diebold, F.X. (2006). Volatility and Correlation Forecasting. Handbook of Economic Forecasting. Editado por G. Elliott., C.W.J. Granger., y A. Timmermann. Amsterdam: North Holland.

[3] Andersen, T.G., y Bollerslev, T. (1998). Answering the Skeptics: Yes, Standard Volatility Models Do Provide Accurate Forecasts. International Economic Review, 39, 885-905.

[4] Akgiray, V. (1989). Conditional Heteroskedasticity in Time Series of Stock Returns: Evidence and Forecasts. Journal of Business, 62, 55-80.

[5] Bates, J.M. y Granger, C.W.J. (1969). The Combination of Forecasts. Operations Research Quarterly, 20, 451-468.

[6] Becker, R. y Clemens, A.E. (2008). Are Combination Forecasts of S\&P 500 Volatility Statistically Superior? International Journal of Forecasting, 24, 122-133.

[7] Benavides, G. (2003). Price Volatility Forecasts for Agricultural Commodities: An Application of Historical Volatility Models, Option Implieds and Composite Approaches for Futures Prices of Corn and Wheat. Working Paper, Lancaster University. url: http://papers.ssrn.com/sol3/papers.cfm?abstract_id $=611062$

[8] Benavides, G. (2006). Volatility Forecasts for the Mexican Peso - US Dollar Exchange Rate: An Empirical Analysis of GARCH, Option Implied and Composite Forecasts Models. Documento de investigación 2006-04, Banco de México. url: http://www.banxico.org.mx/documents/\%7B7F1FAAE1-FEFC-EB00-B0C855420567F74F \%7D.pdf

[9] Black, F. y Scholes, M. S. (1973). The Pricing of Options and Corporate Liabilities. Journal of Political Economy, 81, May-June, 637-654.

[10] Blair, B. J., Poon, S. y Taylor, S. J. (2001). Forecasting S\&P 100 Volatility: The Incremental Information Content of Implied Volatilities and High-Frequency Index Returns. Journal of Econometrics, 105, 5-26.

[11] Bollerslev, T. P. (1986). Generalized Autoregressive Conditional Heteroskedasticity. Journal of Econometrics, 31, 307-327.

[12] Bollerslev, T. P., Engle, R. F. y Nelson D. B. (1994). ARCH Models. Handbook of Econometrics. Vol 4. Editado por R. F. Engle y D. L. McFadden. Amsterdam: Elsevier.

[13] Canina, L. y Figlewski, S. (1993) The Informational Content of Implied Volatility. Review of Financial Studies, 6, 659-681.

[14] Clemen, R. T. (1989). Combining Forecasts: A Review and Annotated Bibliography. International Journal of Forecasting, 5, 559-583. 
[15] Cumby, R., Figlewski, S. y Hasbrouck, J. (1993). Forecasting Volatilities and Correlations with EGARCH Models. Journal of Derivatives, 1, 51-63.

[16] Day, T. E. y Lewis, C. M. (1992). Stock Market Volatility and the Information Content of Stock Index Options. Journal of Econometrics, 52, 267-287.

[17] Deutsch, M., Granger, C.W.J. y Terasvirta, T. (1994). The Combination of Forecasts Using Changing Weights. International Journal of Forecasting, 10, 47-57.

[18] Diebold, F. X. y Mariano, R. S. (1995). Comparing Predictive Accuracy. Journal of Business and Economic Statistics, 13, 253-263.

[19] Ederington, L. y Guan, W. (2002). Is Implied Volatility an Informationally Efficient and Effective Predictor of Future Volatility? Journal of Risk, 4, 3.

[20] Elliott, G. y Timmermann, A. (2005). Optimal Forecast Combination Weights under Regime Switching. International Economic Review, 46, 1081-1102.

[21] Engle, R. F. (1982). Autoregressive Conditional Heteroskedasticity with Estimates of the Variance of U.K. Inflation. Econometrica, 50, 987-1008.

[22] Engle, R.F y Ng, V.K. (1993). Measuring and Testing the Impact of News on Volatility. Journal of Finance, 48, 1749-1778.

[23] Fleming, J. (1998). The Quality of Market Volatility Forecasts Implied by S\&P 100 Index Option Prices. Journal of Empirical Finance, 5, 317-345.

[24] Fleming, J., Ostdiek, B. y Whaley, R.E. (1995). Predicting Stock Market Volatility : A New Measure. Journal of Futures Markets, 15, 265-302.

[25] Garman, M. B y Klass, M. J. (1980). On the Estimation of Security Price Volatilities from Historical Data. Journal of Business, 53, 67-78.

[26] Garman, M.B. y Kohlhagen, S.W. (1983). Foreign Currency Option Values. Journal of International Money and Finance, 2, 231-237.

[27] Giacomini, R. y White, H. (2006). Tests of Conditional Predictive Ability. Econometrica, 74, 1545-1578.

[28] Granger, C.W.J. y Ramanathan, R. (1984). Improved Methods of Combining Forecasts. Journal of Forecasting, 3, 197-204.

[29] Granger, C.W.J. (2003). Long Memory Process-An Economist's Viewpoint. Advances in Statistics, Combinations, and Related Areas, editado por C. Gulats, et al., 100-111. World Scientific Publishers.

[30] Guidolin, M. y Timmermann, A. (2006). Forecasts of US Short-term Interest Rates: A Flexible Forecast Combination Approach. Federal Reserve Bank of St. Louis, Working Paper Series. url: http://research.stlouisfed.org/wp/2005/2005-059.pdf 
[31] Guo, D. (1996). The Predictive Power of Implied Stochastic Variance from Currency Options. Journal of Futures Markets, 16, 915-942.

[32] Hansen, P.R. y Lunde, A. (2005). A Forecast Comparison of Volatility Models: Does Anything Beat a GARCH(1,1)? Journal of Applied Econometrics, 20, 873-889.

[33] Jorion, P. (1995). Predicting Volatility in the Foreign Exchange Market. The Journal of Finance, 50, 507-528.

[34] Lamoureux, C. G. y Lastrapes, W. D. (1993). Forecasting Stock Return Variance: Toward an Understanding of Stochastic Implied Volatilities. The Review of Financial Studies., 6, 293-326.

[35] Manfredo, M. Leuthold, R. M. y Irwin, S. H. (2001). Forecasting Cash Price Volatility of Fed Cattle, Feeder Cattle and Corn: Time Series, Implied Volatility and Composite Approaches. Journal of Agricultural and Applied Economics, 33, 523-538.

[36] Newey, W. y West, K. (1987). A Simple Positive Semi-definite, Heteroskedasticity and Autocorrelation Consistent Covariance Matrix. Econometrica, 55, 703-708.

[37] Parkinson, M. (1980) The Extreme Value Method for Estimating the Variance of the Rate of Return. Journal of Financial Economics, 18, 199-228.

[38] Patton, A. J. (2006) Volatility Forecast Comparison using Imperfect Volatility Proxies. Quantitative Finance Research Centre, University of Technology Sydney, Research Paper 175 .

[39] Patton, A. J. y Sheppard, K. (2007). Evaluating Volatility and Correlation Forecasts. Handbook of Financial Time Series. Editado por T.G. Andersen, R.A., Davis, J.-P. Kreiss y T. Mikosch. Springer Verlag (próximo a publicarse).

[40] Pesaran, M.H. y Timmermann, A. (2007). Selection of Estimation Window in the Presence of Breaks. Journal of Econometrics, 137, 134-161.

[41] Poon, S-H. y Granger, C.W.J. (2003). Forecasting Volatility in Financial Markets: A Review. Journal of Economic Literature, 41, 478-539.

[42] Pong, S., Shackleton, M., Taylor, S. y Xu, X. (2004). Forecasting Currency Volatility: A Comparison of Implied Volatilities and AR(FI)MA Models. Journal of Banking and Finance.

[43] Szakmary, A, Ors, E., Kim, J. K. y Davidson III, W. D. (2003). The Predictive Power of Implied Volatility: Evidence from 35 Futures Markets. Journal of Banking and Finance, $27,2151-2175$.

[44] Taylor, S. J. (1986). Modeling Financial Time Series. Wiley.

[45] Taylor, S. J. (2005). Asset Price Dynamics, Volatility, and Prediction. Princeton University Press. 
[46] Timmermann, A. (2006). Forecast Combinations. Handbook of Economic Forecasting. Editado por G. Elliott., C.W.J. Granger., y A. Timmermann. Amsterdam: North Holland.

[47] West, K.D. (1996). Asymptotic Inference about Predictive Ability. Econometrica, 64, 1067-1084.

[48] Xu, X. y Taylor, S. J. (1995). Conditional Volatility and the Informational Efficiency of the PHLX Currency Options Market. Journal of Banking and Finance, 19, 803-821. 


\section{Cuadro 1. Evaluación de los Pronósticos Individuales}

(2 de enero de 2004 - 31 de diciembre de 2007)

\begin{tabular}{rcc}
\hline Pronóstico & Error Medio & Cociente EPCM \\
\hline \hline GARCHroll6 & $-2.00 \mathrm{E}-06$ & 1,00 \\
GARCHroll3 & $1.24 \mathrm{E}-07$ & 1.06 \\
iv1week & $-7.05 \mathrm{E}-06$ & 1.33 \\
iv3weeks & $-6.79 \mathrm{E}-06$ & 1.19 \\
ivmb & $-5.37 \mathrm{E}-05$ & 155.72 \\
\hline
\end{tabular}

Este cuadro muestra el Error Medio y el cociente del Error de Pronóstico Cuadrático Medio (EPCM) de los modelos del pronóstico de la volatilidad para la volatilidad de los rendimientos diarios spot del tipo de cambio MXN - USD. Los resultados se refieren a pronósticos fuera de la muestra. La volatilidad realizada utilizada es la volatilidad del rendimiento spot intra-día ex-post anualizada.

Las abreviaturas de los modelos son las siguientes: GARCHroll6 y GARCHroll3 denotan el modelo $\operatorname{GARCH}(1,1)$ estimado mediante ventanas móviles de seis y tres años, respectivamente, y una distribución-t. iv1week e iv3week son los modelos con datos duros de volatilidad implícita en opciones con vencimiento a una y tres semanas, respectivamente. ivmb es la volatilidad implícita en opciones en base al modelo con vencimiento a un mes, estimada mediante el modelo de Garman y Kohlhagen (1983). 
Cuadro 2. Prueba Diebold-Mariano-West

(2 de enero de 2004 - 31 de diciembre de 2007)

\begin{tabular}{|c|c|c|c|c|c|}
\hline \multicolumn{6}{|c|}{ Valores-p \Estadístico-t } \\
\hline & GARCHroll6 & GARCHroll3 & iv1week & iv3weeks & ivmb \\
\hline GARCHroll6 & - & -1.57 & -1.99 & -1.84 & -3.82 \\
\hline GARCHroll3 & 0.1164 & - & -1.61 & -1.11 & -3.82 \\
\hline iv1week & 0.0474 & 0.1079 & - & 0.89 & -3.81 \\
\hline iv3weeks & 0.0657 & 0.2679 & 0.3711 & - & -3.81 \\
\hline ivmb & 0.0001 & 0.0001 & 0.0001 & 0.0001 & - \\
\hline
\end{tabular}

El cuadro reporta los resultados de la prueba (en pares) de Diebold-Mariano-West de igualdad de habilidad de predicción utilizando los EPCM como función de pérdida. El estadístico de Diebold-Mariano se presenta arriba de la diagonal y abajo de la diagonal los valores-p correspondientes. En cada caso, la diferencia se calculó como el pronóstico del renglón menos el pronóstico de la columna. Abreviaturas como en el Cuadro 1. 
Cuadro 3. Evaluación de las Combinaciones de los Pronósticos (2 de enero de 2006 - 31 de diciembre de 2007)

\begin{tabular}{rccc}
\hline Combinación & Error Medio & Cociente EPCM & Cociente DMW \\
\hline \hline Ponderaciones iguales & $-1.23 \mathrm{E}-05$ & 6.48 & 0.0028 \\
GR1 & $-1.95 \mathrm{E}-06$ & 0.66 & 0.0266 \\
GR2 & $-2.30 \mathrm{E}-06$ & 0.68 & 0.0337 \\
GR3 & $-4.54 \mathrm{E}-06$ & 0.79 & 0.1664 \\
GARCHroll6 & $-2.29 \mathrm{E}-06$ & 1.00 & $\mathrm{X}$ \\
\hline
\end{tabular}

Este cuadro presenta el Error Medio, el cociente del Error de Pronóstico Cuadrático Medio (EPCM) y los valores-p de las pruebas (en pares) de Diebold-Mariano-West de los modelos de pronóstico de la volatilidad para los rendimientos diarios spot del tipo de cambio MXN-USD. La volatilidad realizada utilizada es la volatilidad del rendimiento spot intradía ex-post anualizada. Todos los pronósticos compuestos incluyen: la volatilidad implícita en base al modelo, la volatilidad implícita con vencimiento a una semana, la volatilidad implícita con vencimiento a tres semanas, el GARCH con ventana móvil de tres años con una distribución-t y el GARCH con ventana móvil de seis años con una distribución-t. Las abreviaturas son las siguientes: Ponderaciones iguales se refiere al promedio simple de los pronósticos. GR = Modelo de tipo Granger-Ramanathan. La primera combinación de GR no incluye una constante y las ponderaciones suman uno, la segunda combinación no tiene una constante, y la tercera combinación tiene una constante y las ponderaciones no son restringidas. GARCHroll6 se refiere al modelo $\operatorname{GARCH}(1,1)$ estimado a partir de una ventana móvil de seis años. 
Cuadro 4. Prueba Giacomini-White

(2 de enero de 2004 - 30 de diciembre de 2005)

\begin{tabular}{rccccc}
\hline \multicolumn{5}{c}{ Valores-p. Instrumentos: Constante $\backslash$ Rezagos de } \\
\hline \multicolumn{5}{c}{ volatilidad realizada, rezagos de $\hat{d}$} \\
GARCHroll6 & - & 0.1380 & 0.0919 & 0.0051 & 0.0098 \\
GARCHroll6 & GARCHroll3 & iv1week & iv3weeks & ivmb \\
iv1week & 0.1915 & - & 0.2600 & 0.0075 & 0.0099 \\
iv3weeks & 0.0013 & 0.5119 & - & 0.0000 & 0.0100 \\
ivmb & 0.0000 & 0.0252 & 0.0000 & - & 0.0101 \\
\hline
\end{tabular}

El cuadro presenta los valores-p de la prueba condicional de habilidad de predicción (en pares) de Giacomini y White (2006). Abajo de la diagonal, el instrumento utilizado es una constante. Arriba de la diagonal los instrumentos son cinco rezagos de la volatilidad realizada y cinco rezagos del diferencial de pérdida. La volatilidad realizada utilizada es la volatilidad del rendimiento spot intra-día ex-post anualizada. Abreviaturas como en el Cuadro 1. 
Cuadro 5. Evaluación de las Combinaciones que Cambian en el Tiempo (2 de enero de 2006 - 31 de diciembre de 2007)

\begin{tabular}{crcccc}
\hline Pronósticos & Combinación & $\begin{array}{l}\text { Error } \\
\text { Medio }\end{array}$ & $\begin{array}{l}\text { Cociente } \\
\text { EPCM }\end{array}$ & DMW $^{\mathrm{a} /}$ & DMW $^{\mathrm{b} /}$ \\
\hline \hline GARCHroll6 & $\begin{array}{r}\text { Híbrido } \\
\text { C }\end{array}$ & $-4.18 \mathrm{E}-06$ & 1.31 & 0.1249 & 0.0502 \\
iv1w & GR1 & $-2.02 \mathrm{E}-06$ & 0.69 & - & 0.1796 \\
\hline GARCHroll3 & Híbrido & $-9.64 \mathrm{E}-07$ & 1.10 & 0.1009 & 0.0254 \\
y & Condicional & $-1.63 \mathrm{E}-06$ & 0.52 & 0.0408 & 0.0705 \\
iv3w & GR1 & $-1.05 \mathrm{E}-06$ & 0.74 & - & $\mathrm{X}$ \\
\hline GARCHroll6 & & $-2.29 \mathrm{E}-06$ & 1.00 & $\mathrm{X}$ & - \\
\hline
\end{tabular}

a/ El punto de referencia es GARCHroll6.

b/ El punto de referencia es GR1.

Este cuadro muestra el Error Medio, el cociente del Error de Pronóstico Cuadrático Medio (EPCM) y los valores-p de las pruebas en pares de Diebold-Mariano-West. Las abreviaturas de los modelos son las siguientes: GARCHroll6, GARCHroll3, iv1week, y iv3week como en el Cuadro 1 y GR1 como en el Cuadro 3. 
Cuadro 6. Evaluación de las Combinaciones que cambian en el Tiempo utilizando Rendimientos Diarios al Cuadrado como Proxy para la Volatilidad Diaria

\begin{tabular}{clllll}
\hline \multicolumn{5}{c}{ (2 de enero de 2006 - 31 de diciembre de 2007) } \\
\hline Pronósticos & Combinación & $\begin{array}{c}\text { Error } \\
\text { Medio }\end{array}$ & $\begin{array}{c}\text { Cociente } \\
\text { EPCM }\end{array}$ & DMW $^{\mathrm{a} /}$ & DMW $^{\mathrm{b} /}$ \\
& & $-5.11 \mathrm{E}-06$ & 0.95 & 0.5562 & \\
\hline \hline GARCHroll3 & Híbrido & -0.0288 \\
$\mathbf{y}$ & Condicional & $-8.61 \mathrm{E}-07$ & 0.80 & 0.1014 & 0.4127 \\
iv1w & GR1 & $-8.01 \mathrm{E}-07$ & 0.85 & - & $\mathrm{X}$ \\
GARCHroll3 & Híbrido & $-1.42 \mathrm{E}-07$ & 1.01 & 0.2134 & 0.0984 \\
y & Condicional & $9.33 \mathrm{E}-07$ & 0.83 & 0.0619 & 0.1200 \\
iv3w & GR1 & $9.96 \mathrm{E}-07$ & 0.92 & - & $\mathrm{X}$ \\
GARCHroll3 & & $8.72 \mathrm{E}-08$ & 1.00 & $\mathrm{X}$ & - \\
\hline
\end{tabular}

Notas y abreviaturas como en el Cuadro 5, con la excepción de que en este cuadro el punto de referencia de modelos individuales es el GARCHroll3. 


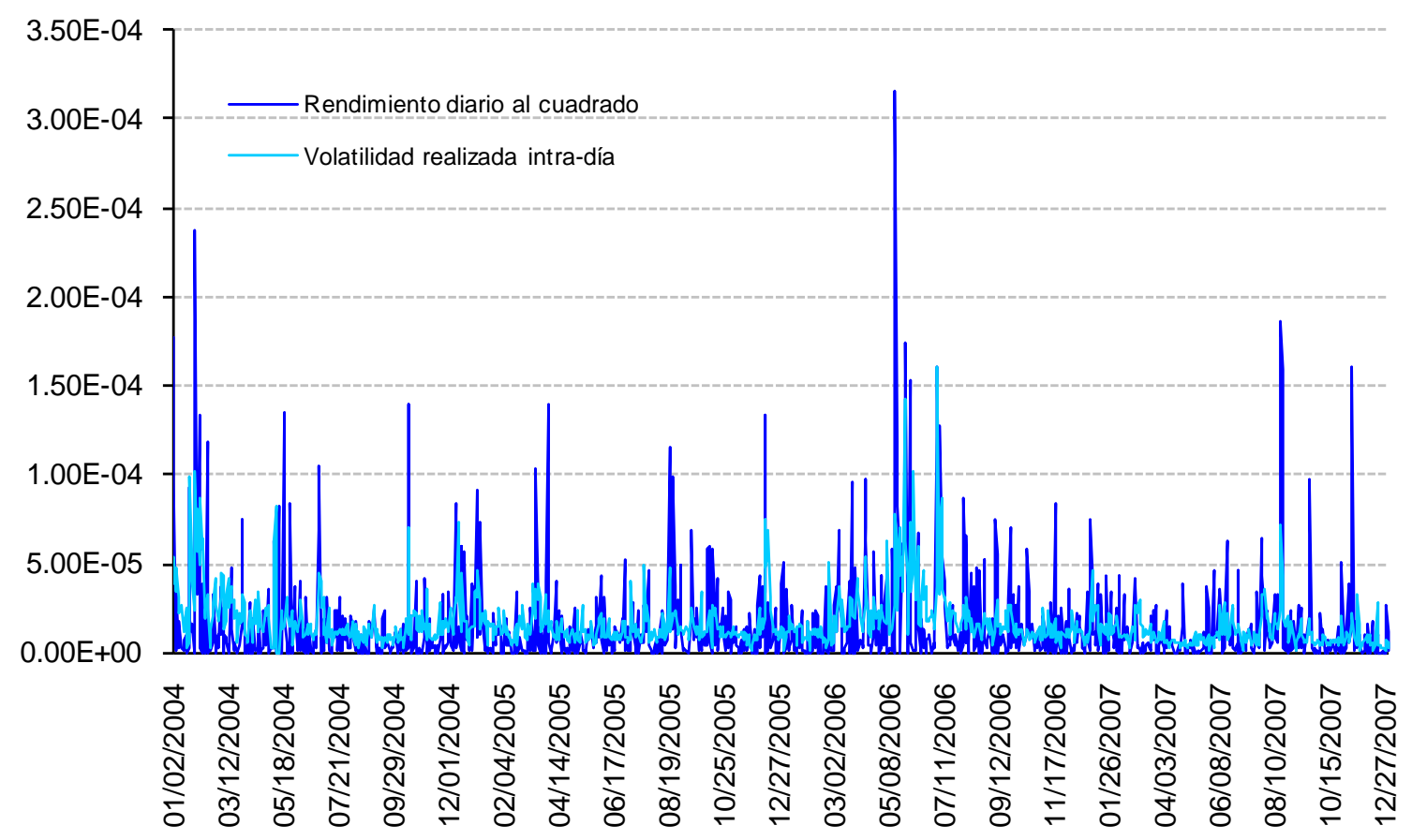

Gráfica 1: Rendimientos diarios al cuadrado y volatilidad realizada intra-día del tipo de cambio MXN-USD. 

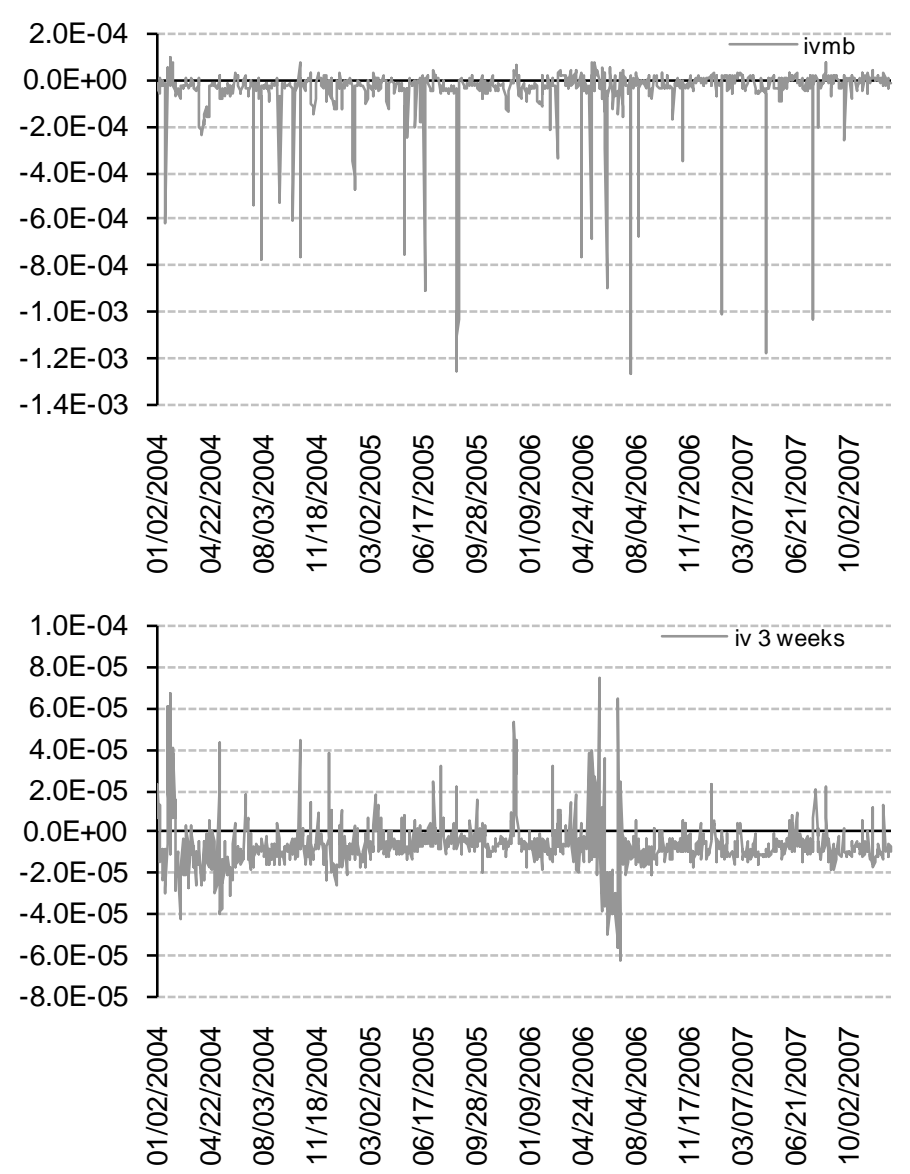
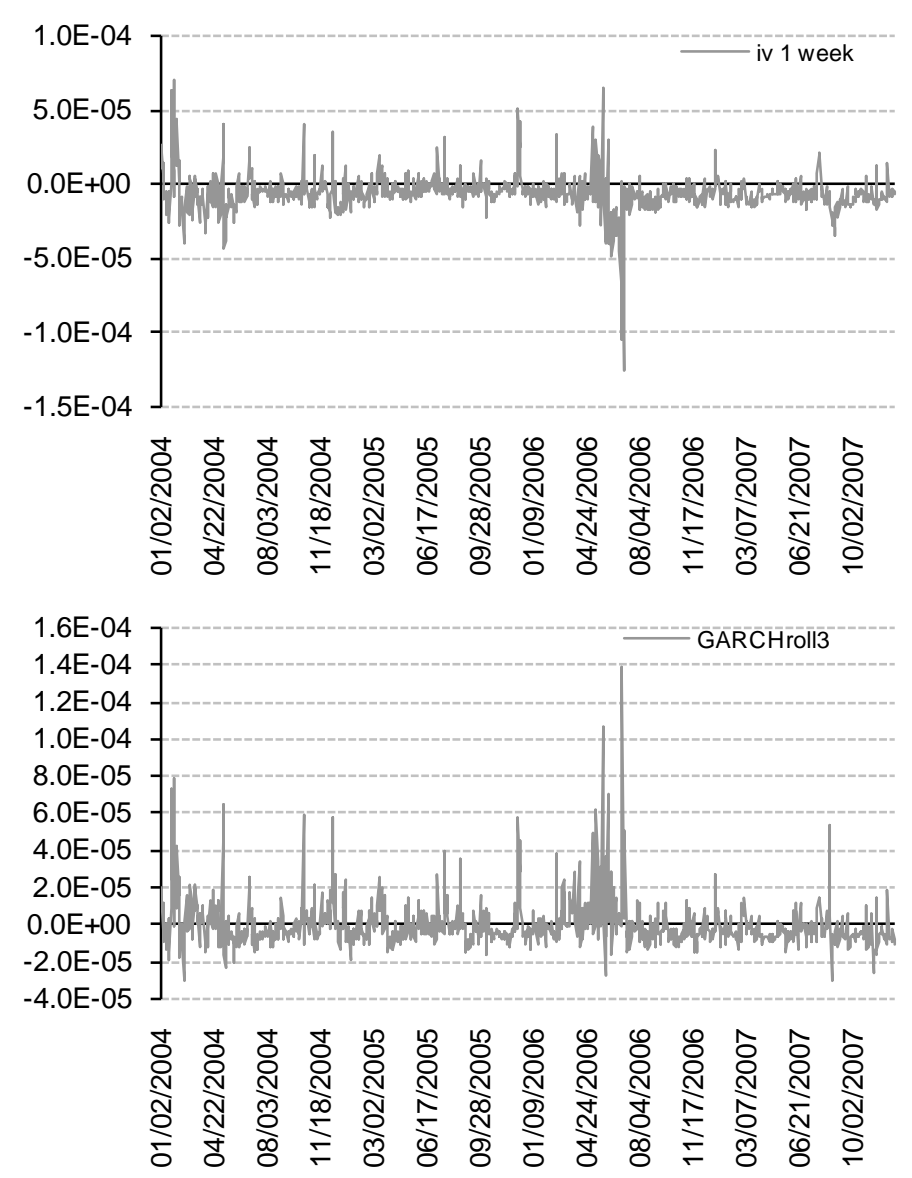

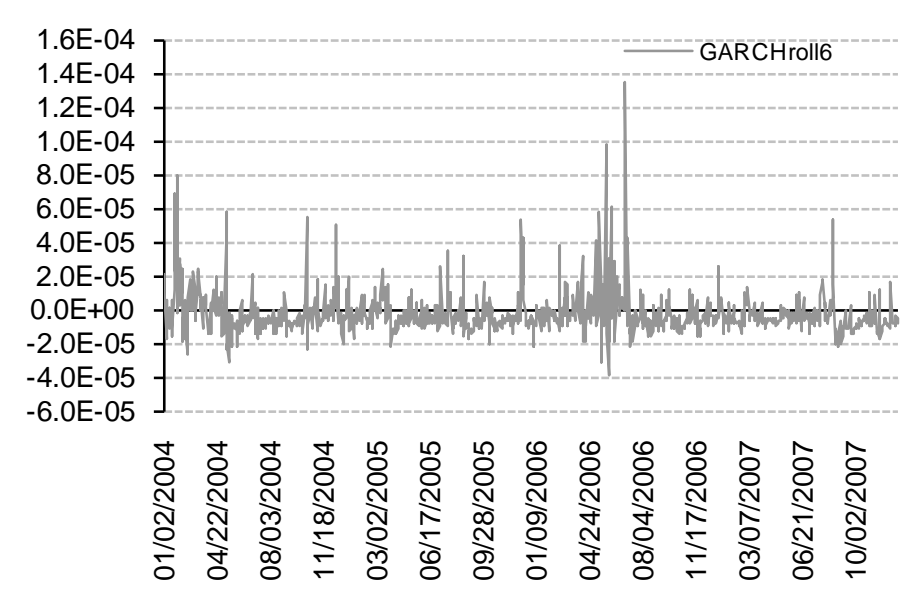

Gráfica 2: Errores de pronóstico de los modelos individuales. 


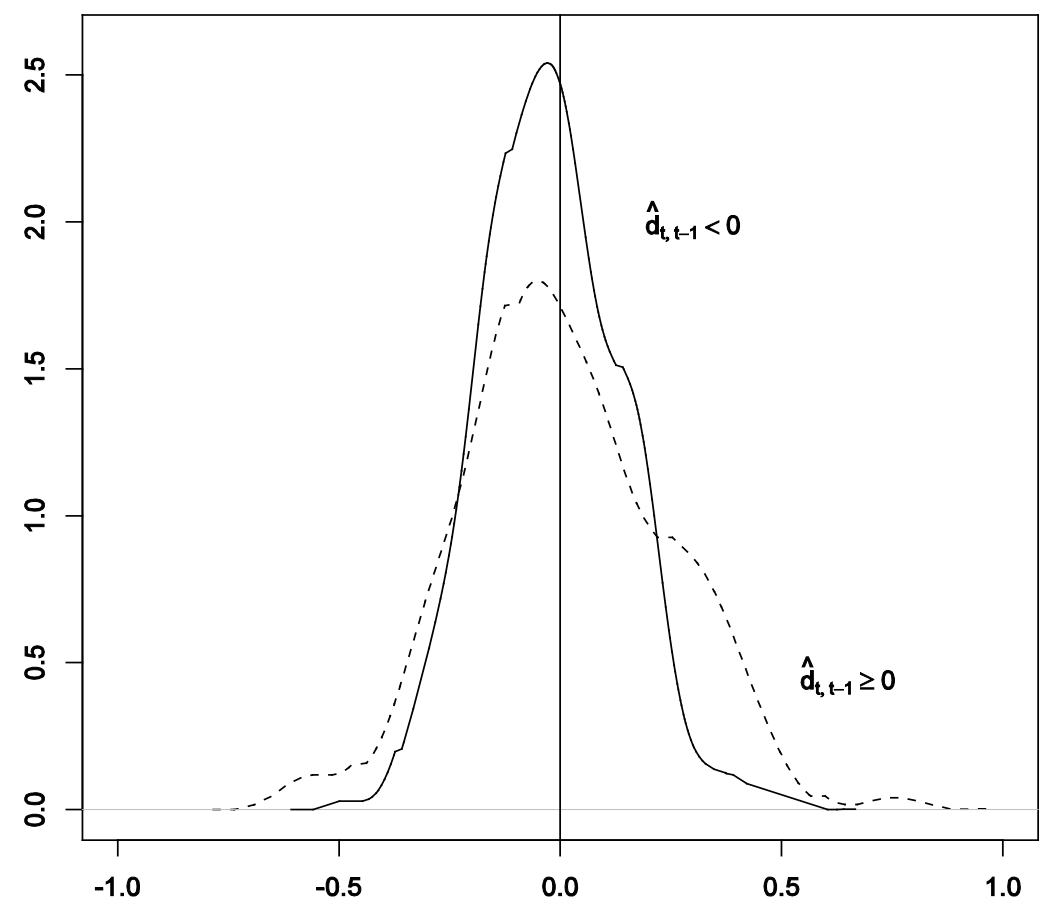

Gráfica 3. Función indicador por rendimientos diarios 2 de enero de 2006 - 31 de diciembre de 2007. 


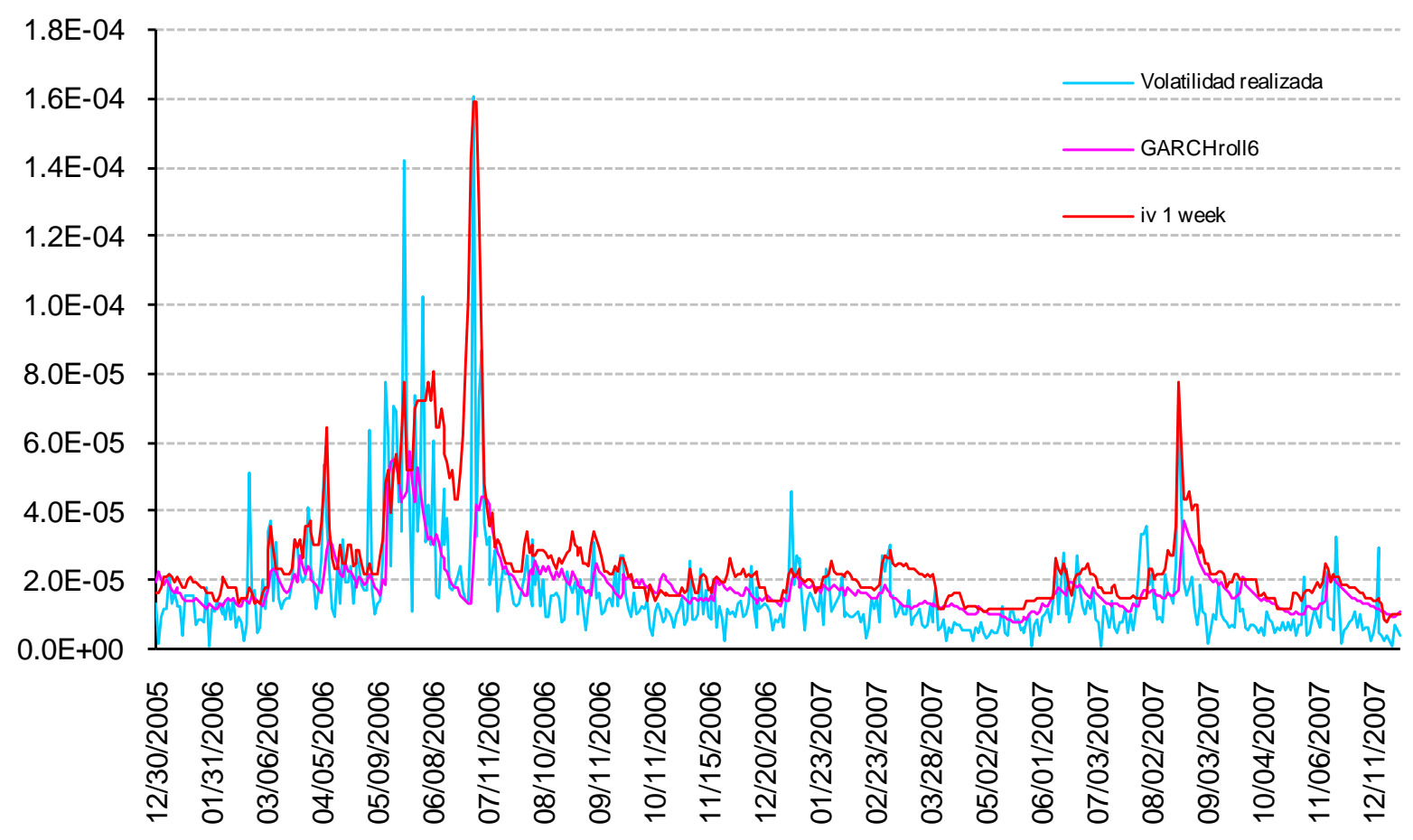

Gráfica 4. Volatilidad realizada y pronósticos del modelo GARCH con ventanas móviles y del modelo de volatilidad implícita en opciones de una semana basado en datos duros. 